\title{
Six-gene Signature Based on Metabolism Is Closely Related to the Prognosis and Immune Infiltration of Patients With Sepsis
}

\section{Huaguo Feng}

Department of Hepatobiliary Surgery and Chongqing Key Laboratory of Hepatobiliary Surgery, the Second Affiliated Hospital of Chongqing Medical University, Chongqing,

\section{Qingbo Pan}

Department of Infectious Diseases, the Second Affiliated Hospital of Chongqing Medical University

\section{Yanqin Wang}

Department of Neurology, Children's Hospital of Chongqing Medical University. Ministry of Education Key Laboratory of Child Development and Disorders. China International Science and Technology Cooperation Base of Child Development and Critical Disorders,

\section{Wenfeng Zhang}

Department of Hepatobiliary Surgery and Chongqing Key Laboratory of Hepatobiliary Surgery, the Second Affiliated Hospital of Chongqing Medical University, Chongqing

\section{Jianping Gong}

Department of Hepatobiliary Surgery and Chongqing Key Laboratory of Hepatobiliary Surgery, the Second Affiliated Hospital of Chongqing Medical University, Chongqing

\section{Fanbo Qin ( $543692640 @ q q . c o m$ )}

Department of Hepatobiliary Surgery and Chongqing Key Laboratory of Hepatobiliary Surgery, the Second Affiliated Hospital of Chongqing Medical University, Chongqing, https://orcid.org/0000-00017180-9550

\section{Research}

Keywords: Sepsis, metabolism associated genes(MAGs), prognosis, immunity

Posted Date: January 29th, 2021

DOI: https://doi.org/10.21203/rs.3.rs-154347/v1

License: (c) (i) This work is licensed under a Creative Commons Attribution 4.0 International License. Read Full License 
Six-gene signature based on metabolism is closely related to the prognosis and immune infiltration of patients with sepsis

\section{Huaguo Feng', Qingbo Pan², Yanqin Wang ${ }^{3}$, Wenfeng Zhang', Jianping Gong1, Fanbo Qin ${ }^{1 *}$}

1 Department of Hepatobiliary Surgery and Chongqing Key Laboratory of Hepatobiliary Surgery, the Second Affiliated Hospital of Chongqing Medical University, Chongqing, 400010, China.

2 Department of Infectious Diseases, the Second Affiliated Hospital of Chongqing Medical University, Chongqing, 400010, China.

3 Department of Neurology, Children's Hospital of Chongqing Medical University. Ministry of Education Key Laboratory of Child Development and Disorders. China International Science and Technology Cooperation Base of Child Development and Critical Disorders, Chongqing Key Laboratory of Pediatrics. National Clinical Research Center for Child Health and Disorders, Chongqing, 400014 China

\footnotetext{
* Corresponding Author: Fanbo Qin

Address: 76\# Linjiang Road, Chongqing, 400010, China. Tel: +86-023-63693532. Fax: +86-023-63693532. Email: 543692640@qq.com
} 


\section{Abstract}

Background: Sepsis is a leading cause of mortality and morbidity in the intensive care unit. Current studies indicated that metabolism-associated genes (MAGs) have critical roles in sepsis. This study aims to construct a gene signature for sepsis and explore its possible mechanisms.

Methods: Differentially expressed metabolism-associated genes (DEMAGs) were identified in sepsis patients based on the Gene Expression Omnibus (GEO) database. Univariate and multivariate Cox regression analyses were performed to identify and construct the prognostic gene signature. Quantitative real-time PCR was applied to examine the mRNA level of signature genes in the sepsis mice model established by cecal ligation and puncture (CLP). Next, Gene Set Enrichment Analysis (GSEA) was performed to further understand the underlying molecular mechanisms of gene signature. We then assessed the abundance of infiltrating immune cells in each sample to explore the immune microenvironment of sepsis patients. Finally, the Tumor Immune Dysfunction and Exclusion (TIDE) and SubMap algorithms were used to explore the immunotherapy response of sepsis patients.

Results: A novel six-gene signature (including ELANE, NQO2, TLR2, PTGDS, SMAD3, CD3E) was established for sepsis prognosis prediction, which could accurately predict the overall survival (OS) of sepsis patients. In the sepsis mice model, except PTGDS, the mRNA expression of the other five genes was consistent with that of sepsis patients. The results of GSEA analysis indicate that the prognostic signature was closely related to immunity function. Besides, we found that the risk score was strikingly negatively correlated with the tumor microenvironment (TME) immune cells infiltration and expression of critical immune checkpoints. The patients in the low-risk group were more likely to benefit from immune therapy through the TIDE and SubMap analysis.

Conclusion: In conclusion, our signature can predict the OS of sepsis patients and provide potential guidance for exploring patients who may benefit from immunotherapy.

Keywords: Sepsis, metabolism-associated genes (MAGs), prognosis, immunity.

\section{Background}

Sepsis is a syndrome of the immune system out of control caused by infection, which can be accompanied by multiple organ failure and systemic inflammatory response [1,2]. The incidence of sepsis is high, with more than 18 million cases of severe sepsis worldwide each year, and this number is increasing at a rate of $1.5 \%-8.0 \%$ per year $[3,4]$. The mortality rate of sepsis has 
surpassed that of myocardial infarction as the leading cause of death among non-cardiac patients in the intensive care unit. With advances in critical care treatment technology, the hospital mortality rate of sepsis patients is still as high as $17 \%$, although it has decreased significantly[4-6]. Therefore, early diagnosis of sepsis and its effective prevention are the keys to improve patient survival.

The metabolic process is necessary to maintain life, including lipid metabolism, glucose metabolism, amino acid metabolism and so on. A growing body of research suggests that changes in metabolic processes are a hallmark of sepsis[7, 8], especially the changes of glucose and lipid metabolism that play important roles in sepsis[9, 10]. In general, glucose metabolism mainly affects the function of immune cells, while lipid metabolism affects the homeostasis of plasma lipoproteins, both of which have a significant influence on the progression and survival of sepsis patients. PKM2 is a key gene in glycolysis pathway, which affects the function of immune cells by regulating Warburg effect and plays an important role in sepsis[11]. Pharmacological inhibition of PKM2-related pathways has been shown to protect mice from fatal sepsis[12]. Studies have shown that the up-regulation of PCSK9, a gene involved in cholesterol metabolism, is associated with acute organ failure in sepsis[13, 14]. The high expression of PCSK9 in sepsis reduces the clearance of pathogen lipids, and PCSK9 inhibition may be an effective strategy in the treatment of sepsis[15]. These genes, which are associate with metabolic processes, are key markers of sepsis progression and treatment. However, the prognostic value and potential mechanisms of metabolism associated genes (MAGs) in sepsis has not been fully explored.

In this study, we screened the DEMAGs based on the GEO database and evaluated the prognostic value of these genes. In addition, we analyzed the biological functions related to prognosis by GSEA to further analyze the immune microenvironment and immunotherapy in sepsis. Furthermore, we further validated the expression of prognosis signature in sepsis mice model by RT-qPCR. These results provide a research direction for exploring the molecular mechanism of MAGs in immunotherapy of sepsis.

\section{Material and methods}

\section{Data collection}

The transcriptome array data of sepsis patients were downloaded from the GEO database, inlcuding the GSE69528 and GSE65682 datasets. The GSE69528 dataset comprised of 111 
samples, including 28 normal and 83 sepsis samples. The GSE65682 dataset contained 802 samples, including 42 normal and 760 sepsis samples. After removing 292 patients from the GSE65682 dataset due to a lack of survival data, a total of 468 sepsis patients and their information were utilized to identify prognostic DEMAGs, which was used to built a prognostic risk model. We downloaded the gene set (h.all.v7.2.entrez.gmt) from the GSEA website (https://www.gsea-msigdb.org/gsea/index.jsp), and a total of 1811 MAGs were obtained from metabolism-associated pathways by enrichment analysis. The detailed MAGs were listed in

\section{Supplementary materials Table S1.}

\section{Identification of differentially expressed genes (DEGs)}

The DEGs were determined between normal and sepsis samples in the GSE65682 and GSE69528 datasets using the 'limma' R package. The $\mid \log _{2}$ fold change (FC) $\mid>1$ and $P$-value $<0.05$ were regarded as the cut-off criteria. Volcano plot was drawn to visualize the differences in gene expression levels between the normal and sepsis samples. After overlapping with 1811 MAGs, we obtained the 75 DEMAGs.

\section{Construction and verification of the prognostic signature}

We randomly divided patients (648 samples) of GSE65682 datasets randomly into a training set $(n=328)$ and a validation set $(n=140)$ at a ratio of 7:3. The univariate Cox regression model was used to identify DEMAGs that were significantly associated $(P<0.05)$ with the OS of the sepsis patients. Subsequently, the candidate DEMAGs were subjected to multivariate Cox regression analysis to evaluate their contribution as prognostic factors in patient survival. The risk score calculating formula was:

Risk score $=$ ExpGene $1 *$ Coef $1+$ ExpGene $2 *$ Coef $2+$ ExpGene $3 *$ Coef $3 \ldots$ where Coef means the regression coefficients of genes, Exp is the normalized expression values of each gene signature. To stratify patients into high- and low-risk groups, the optimum cutoff value for the risk score was determined using the 'survminer' package in R. Next, the K-M survival curve and log-rank test were performed to evaluate the OS between high- and low-risk groups. The area under the curve (AUC) of receiver operating characteristic (ROC) was calculated using the 'survival ROC' package in R. In addition, the risk plot was illustrated using the 'pheatmap' package in R.

\section{Functional enrichment analysis (GSEA)}

The 468 sepsis patients of the GSE65682 dataset were divided into high- and low-risk groups 
based on the median value of risk score. Then, we performed GSEA on all genes between the two risk groups using the clusterProfiler of R. Biological functions of GO and KEGG terms with the threshold of $P$-value $<0.05$ was considered as significant.

\section{Evaluation of immune microenvironment and immunotherapy efficiency}

The immune-, stromal- and estimate scores from each sepsis patients were calculated by applying the 'Estimation of Stromal and Immune cells in MAlignant Tumours using Expression data' (ESTIMATE) algorithm. Spearman correlation analysis was used to evaluate the correlation between risk score and the immune-, stromal- and estimate scores, respectively. Microenvironment cell population-counter (MCP-counter), a methodology based on gene expression profile data, was used to evaluate absolute abundance of 8 immune and 2 nonimmune stromal cell populations. In addition, the SubMap analysis was applied to predict the clinical response to the immune checkpoint blockade (CTLA-4, PD-1 and PD-L1). TIDE (http://tide.dfci.harvard.edu/) was used to estimate TIDE prediction scores with normalized transcriptome data from each patient. A low TIDE prediction score represents weak potential immune escape, and these patients would potentially exhibit a greater immune therapy response[16]. Thus, TIDE was used to predict the efficacy of immunotherapy in two risk groups.

\section{Quantitative real-time-PCR validation}

Sepsis mice model constructed by cecal ligation and puncture (CLP) to validate the expression level of prognostic signature genes. Male, 6 8 weeks old, C57BL/6 mice weighing 18 20g were used for experiments (Brought from Experiments Animal Center of Chongqing Medical University). The mice were maintained in accordance with the National Institutes of Health guidelines for animal research and the legal requirements in China. The mice were randomized into a sham control group $(n=5)$ and a CLP group $(n=5)$. Twelve hours after the operation, the mice were sacrificed and peripheral blood tissues were harvested. Total RNA was extracted using TRIzol (Takara, Dalian, China), and reverse transcription was performed using RT reagent Kit with gDNA Eraser (Takara, Japan). Reactions were performed in a Bio-Rad CFX Connect TM Real-Time System (Bio-Rad, Hercules, CA, USA). The following qPCR primer sequences were used: $\beta$-actin forward primer 5'-CCCATCTACGAGGGCTAT-3'; $\beta$-actin reverse primer 5'-ATGTCACGCACGATTTCC-3'; NQO2 forward primer 5'-AAAGTGCTCATCGTCTA-3'; NQO2 reverse primer 5'-CCTGCTTGCTCAGTTCT-3'; ELANE forward primer 
5'-GTGCCACCCTCATTGCC-3'; ELANE reverse primer 5'-TGTCGAGTGCGCTCCTG-3'; TLR2 forward primer 5'-GATGGTGAAGGTTGGAC-3'; TLR2 reverse primer 5'-AGGTTTCGGTAAGTTGT-3'; CD3E forward primer 5'-TGTGACCCGAGGAACCG-3'; CD3E reverse primer 5'-TGCTCGGAGGGCTGGAT-3'; PTGDS forward primer 5'-CATGCTGTGGATGGGTTTGG-3'; PTGDS reverse primer 5'-GCTTGAGTTGGAGGCG AGGC-3'; SMAD3 forward primer 5'-GGCTTTGAGGCTGTCTACCA-3'; SMAD3 reverse primer 5'-CCCAACCCGCTCCCTTTACT-3'.

\section{Statistical analysis}

Statistical analysis was performed using the $\mathrm{R}$ programming language and GraphPad Prism version 8.0.2 software. Student's t-test was used to analyze real-time gene expression analysis. In all analyses, $P$-value less than 0.05 were considered as statistically significant.

\section{Results}

\section{Identification of DEMAGs in Sepsis}

To identify genes that may play critical roles in the development and progression of sepsis, the datasets (GSE65682 and GSE69528) were analyzed respectively to identify DEGs between normal and sepsis samples. A total of 1165 DEGs were identified in the GSE65682 dataset, of which 400 were upregulated and 765 were downregulated (Fig. 1A, Supplementary materials Table S2). For the GSE69528 dataset, 1377 DEGs were identified, including 644 upregulated and 733 downregulated genes (Fig.1B, Supplementary materials Table S3). We further identified 75 DEMAGs from the intersection of the 1811 MAGs and DEGs in the GSE65682 and GSE69528 datasets (Fig.1C, Supplementary materials Table S4).

\section{Selection of prognosis-related DEMAGs}

We conducted a univariate Cox regression to investigate the correlation of the 75 DEMAGs with the OS of sepsis patients in the GSE65682 training set and identified 15 DEMAGs significantly related to OS in sepsis patients (Fig.2A, Supplementary materials Table S5, $P<0.05$ ). Subsequently, we conducted a stepwise multivariate Cox regression analysis. Six of the 15 DEMAGs were extracted and considered as significantly connection with the OS of sepsis patients. Forest plot showed ELANE was associated with poor outcomes, and NQO2, TLR2, PTGDS, SMAD3 and CD3E were associated with favorable outcomes (Fig.2B, Supplementary materials Table S6, $P<0.05)$. 


\section{Evaluation and validation of the prognostic risk model}

We calculated the prognostic risk score for each patient in the training set. Patients were divided into the high- and low-risk groups according to the median value of risk score. K-M survival analysis demonstrated that patients with high risk scores had significantly poorer OS than patients with low risk scores (Fig.3A, $P<0.0001)$. We drew a ROC curve to indicate the accuracy of the risk model, and the AUC values of the risk model for 28 days was 0.696, which indicated that the risk model had good sensitivity for survival prediction (Fig.3B). The survival status of the patients and the expression of the 6 DEMAGs were shown in Fig.3C-E. With the increase of risk score, the mortality rate of patients in the high-risk group was significantly higher than that in the low-risk group. To validate the prognostic value of the 6-gene signature, the patients of validation set was divided into high- and low-risk groups according to the same cut-off value of training set, and the results were consistent with the training set, as shown in Fig.3F-J. Taken together, these data suggest that the 6 key DEMAGs could effectively predict the prognosis of sepsis patients.

\section{Identification of the gene signature-related biological processes and pathways}

The GSEA analysis was used to elucidate the biological functions and pathways associated with the 6 prognostic signature. The results revealed that numerous immune-related GO terms were significantly enriched, which included activation of immune response, adaptive immune response, antigen receptor mediated signaling pathway and immune receoptor activity, etc. Top 10 biological functions were shown in Fig.4A-B and Supplementary materials Table S7). The KEGG analysis revealed that significant enrichment pathway were Natural killer cell mediated cytotoxicity, $\mathrm{T}$ cell receptor signaling pathway, and Antigen processing and presentation (Fig.4C-D and Supplementary materials Table S8). All these demonstrated the prognostic signature is highly positive associated with the immune related biological pathway.

\section{Comparison of the immune microenvironment between high- and low-risk groups}

In order to evaluate the discrepancy of immune microenviroment between the high- and low-risk groups, the stromal-, immune-, and estimate scores were compared between high- and low-risk groups. The results showed that stromal-, immune-, and estimate scores in the high-risk group were significantly lower than that in the low-risk group (Fig.5A-C). At the same time, we found that risk score has a negative correlation with immune- and estimate scores (Fig.5D). Furthermore, we analyzed the proportion of 10 immune cells in the high- and low-risk groups, as shown in 
Fig.5E-F. The proportion of multiple immune cells were remarkable higher in low-risk group including T cells, CD8 T cells, Cytotoxic lymphocytes, B lineage, NK cells, Monocytic lineage, Neutrophils. Instead, Myeloid dendritic cells, Endothelial cells, and Fibroblasts proportion were lower in low-risk group. These findings suggested that there were significant differences of immune microenviroment between the two risk groups.

\section{Evaluating the therapeutic response of the high- and low-risk groups}

Immune checkpoint molecules are essential for immune function and have diverse clinical implications in immunotherapy. Therefore, we contrasted the expression of immune checkpoint molecules between the two risk groups. We determined the expression of several key immune checkpoint molecules, including IDO1, PD-L1 (CD274), PD-L2 (PDCD1LG2), TIM-3 (HAVCR2), TIGIT, PD-1 (PDCD1), LAG3, ICOS, and CD27. As shown in Fig.6A, the expression of immune checkpoint molecules in the low-risk group was greater than that in the high-risk group, except PD-1 and PD-L2.

Immune checkpoint receptors including programmed death-1 (PD-1) and cytotoxic $\mathrm{T}$ lymphocyte antigen-4 (CTLA-4) have been shown to be increased on immune cells during sepsis and hypothesized to be one of the major contributors causing sepsis induced immune cell dysfunction[17]. Therefore, we used the SubMap algorithm to estimate the clinical response of the high- and low-risk groups to immune checkpoint blockade (CTLA-4 and PD-1). Interestingly, we found that the low-risk group patients had a sensitive respond for anti-PD-1 therapy $(P<0.05$; Fig.6B). In addition, the TIDE algorithm was utilized to calculate the immunotherapy response rates in the two risk groups. And the TIDE value was significantly higher in the high-risk group compared with that in low-risk group, indicating that patients with low risk score had more sensitive to respond to immune therapy $(P<0.05 ;$ Fig.6C).

\section{Experimental verification of 6 DEMAGs in mRNA level}

To confirm the reliability of the bioinformatics analysis, we validated the expression level of the six DEMAGs in sepsis mice model by quantitative real-time PCR. Consistent with the results of bioinformatics analysis, the expression of NQO2, ELANE and TLR2 were significantly upregulated in the(CPL), while the expression of $\mathrm{CD} 3 \mathrm{E}$ and SMAD3 were significantly downregulated in CPL group. The expression of PTGDS was not consistent with the results of bioinformatics analysis, which showed upregulated in CPL group (Fig.7A). In addition, the 
expression of the six genes in GSE65682 and GSE69528 are shown in the figure (Fig.7B-C).

\section{Discussion}

Despite advances in treatment strategies for sepsis in recent years, it remains a leading cause of death worldwide due to the absence of specific treatments and drugs[3]. The pathologic process of sepsis is complex and subject to the interplay of multiple pathways, such as hypoxia[18], immune activation, and changes of metabolic processes[19]. Among them, the changes of metabolic processes on sepsis has been well understood in recent years. Therefore, it seems logical to develop new predictive or therapeutic targets based on genes involve in metabolic processes. In this study, we focused on the MAGs and firstly constructed a six-gene signature in sepsis. Then, we demonstrated that the 6-gene signature has a good predictive value for patient prognosis, immune microenvironment and responsiveness to immunotherapy. This study has some implications for the mechanism of sepsis research and clinical practice.

In the present study, combining the two independent GEO datasets and metabolism associated gene set, we obtained a 6-gene signature (including ELANE, NQO2, TLR2, PTGDS, SMAD3, CD3E). In the 6-gene signature, ELANE, NQO2 and TLR2 were significantly up-regulated, while PTGDS, SMAD3 and CD3E were significantly down-regulated in sepsis. All the above results were verified in the sepsis mice model. The expression levels of five genes, ElANE, NQO2, TLR2, CD3E and SMAD3, in sepsis mice model were consistent with in sepsis patients. However, the expression level of PTGDS was contrary to our silico mRNA expression in sepsis patients. This result suggests that PTGDS may play different roles in sepsis patients and mice model. In addition, we found that of these six genes, ELANE was associated with poor outcomes, while the remaining five genes were associated with favorable outcomes. Combined with previous studies, we found that ELANE[20-22], TLR2[23-25], and SMAD3[26, 27] have been clearly reported in sepsis, while the roles of CD3E, NQO2 and PTGDS in sepsis remain unclear. Previous studies have shown that the expression of ELANE is able to reflect the leukocyte function during sepsis, which is able to provide more prognostic information than leukocyte counting[21]. Brittney Williams et al found that TLR2 and TLR7 are involved in coagulation dysfunction in murine sepsis, which may be an important reason for the high mortality of sepsis[28].The regulatory axis of GDF / SMAD2 / SMAD3 in sepsis that can promote macrophage polarization toward M2 phenotype leading to immunosuppression, and activation of this regulatory axis may be associated with high 
lethality in sepsis[26]. CD3E, as part of the TCR-CD3 complex, is present on the surface of T-lymphocytes and plays an important role in adaptive immunity[29]. In agreement with the findings of Raquel Almansa et al., the expression of CD3E was inversely associated with sepsis mortality[30]. The related research reports on PTGDS showed that PTGDS was mainly related to nervous system diseases[31, 32]. In the inflammatory response, PTGDS is involved in eicosanoid $\begin{array}{lllll}\text { synthesis and achidonic } & \text { acid }\end{array}$ (N-Ribosyldihydronicotinamide:Quinone Reductase 2) encodes a member of the thioredoxin family of enzymes and diseases associated with NQO2 include breast cancer[34-36] and alcoholic pancreatitis[36]. Previous studies[37] have shown that NQO1 and NQO2 combined protect mice against lung inflammation as well as lung injury induced by hyperoxia, which was consistent with our result that NQO2 was a protective factor (Hazard ratio=0.59) for sepsis patients.

Sepsis is characterized by the immune system out of control, specifically the early over activation of the immune system and subsequent immunosuppression[38]. In fact, studies have shown that more than $60 \%$ of patients die in the immunosuppressive stage of sepsis, consistent with defective host immunity[38, 39]. In the present study, we divided the patients into high- or low- risk groups based on the score of the six-gene signature, and performed GSEA enrichment analysis to explore the biological function and pathways related to the prognostic signature. Interestingly, the results suggested that prognostic signature was significantly associated with immune-related biological functions. The results of GSEA enrichment analysis are consistent with conclusion of previous studies, that is, immunosuppression in sepsis is closely related to the risk of poor outcomes [38]. In addition to this, we found that the immune microenvironment of sepsis patient in high-risk and low-risk groups was significantly different, and the composition of immune cells was significantly correlated with the risk score. The results of MCP-counter further distinguished the different types of immune cells in each group. Consistent with previous studies, we found that the proportion of $\mathrm{T}$ cells[40], B cells[41, 42], NK cells[43] and neutrophils[44] was significantly lower in patients with higher risk. We speculate that this phenomenon may also be closely related to the immunosuppression of sepsis. In addition, the proportion of myeloid dendritic cells, endothelial cells and fibroblasts was higher in the high-risk group. Among the three types of cells, the role of myeloid dendritic cells in sepsis has been poorly studied, and the functions of endothelial cells[45, 46] and fibroblasts[47] have only been partially explored. Therefore, the changes in the proportion 
of these immune cells in different prognostic septic patient need to be further explored.

Based on the conclusion that there was significant differences of immune microenvironment between high- and low-risk groups, we further explored the responsiveness of the two risk groups to immunotherapy. We compared the expression levels of some immune checkpoint molecules commonly used in the field of tumor immunotherapy, hoping to provide enlightenment for immunotherapy of sepsis[48]. The results shown that the expression levels of PD-1 and PD-L2 were significantly up-regulated in the high-risk group, which was contrary to the trend of CD27, HAVCR2, ICOS, LAG3 and TIGIT. Immunosuppression is considered to be an important cause of high mortality in sepsis[49]. Numerous studies have shown that immune checkpoint molecules in sepsis are significantly upregulated, which may be an important reason for immunosuppression[48-50]. The regulatory axis formed by PD-1 and its ligand PD-L1 / PD-L2 is the most characteristic interaction in immunopathology of sepsis. In the field of sepsis treatment, there are also an increasing number of preclinical and clinical studies showing that targeting both PD-1 and PD-L1 can improve host anti infectivity[51-54]. Recently, the first clinical evaluation of a PD-1/PD-L1 inhibitor showed that the drug was well tolerated, found no evidence of cytokine storm and showed signs of recovery of immune status within 28 days at high doses[55, 56]. In addition, studies have shown that LAG3 can resist the role of PD-1 in blocking tumor therapy[57], and Niu et al. also observed the opposite expression trend of LAG3 and PD-1 in sepsis and its important role in $\mathrm{T}$ cell exhaustion[58]. However, the role of the other immunocheckpoint molecules plays in the immunotherapy of sepsis are still limited and needs to be further explored. Furthermore, the results of TIDE and SubMap algorithm also showed that low-risk group may be more sensitive to immune checkpoint blockade treatment. Interestingly, we found that although the expression of PD-1 was lower in the low-risk group, while this group was more sensitive to the treatment of PD-1 inhibitor. Thus, we speculated that the expression of immune checkpoint molecules may not be directly related to the sensitivity of immunotherapy to this molecule, and the correlation between them remains to be further explored.

In this study, we successfully constructed a 6-gene signature based on MAGs. The signature could be used to predict survival and responsiveness to immunotherapy of sepsis, which may contribute to the development or optimization of new treatment for sepsis. However, there are some limitations in our study. Firstly, TIDE is an algorithm developed based on the data of melanoma 
and non-small cell lung cancer to predict anti-PD-1 and anti-CTLA4 treatment reactivity, which may produce bias when applied to sepsis[16, 59]. However, considering that the mechanism of immune escape in tumor is similar to the mechanism of immune suppression in sepsis, and there is no better method to predict the response of sepsis immunotherapy to our best knowledge, we apply this algorithm to sepsis in order to make some exploratory contributions to the field of immunotherapy of non-neoplastic diseases [60-62]. Secondly, we only verified the expression of these six genes in the sepsis mice model, and the potential biological mechanism remains to be further explored. Thirdly, other types of sepsis, such as urinary tract infection or purulent meningitis, were not included in this study due to limited dataset. These parts of patients not included in the study need further analysis.

\section{Conclusion}

In conclusion, we identified and validated a prognostic signature for patients with sepsis, which likely reflects the immune dysregulation in the tumor microenvironment and is a potential prognostic biomarker and therapeutic target.

\section{Abbreviations}

MAGs: Metabolism-associated genes; GEO: Gene Expression Omnibus; DEMAGs: Differentially expressed metabolism-associated genes; CLP: Ligation and puncture; GSEA: Gene Set Enrichment Analysis; TIDE: Tumor Immune Dysfunction and Exclusion; ROC: Receiver operating characteristic; AUC: Area under the curve.

\section{Acknowledgements}

None

\section{Authors' contributions}

Fanbo Qin conceived the idea, performed data acquisition and analysis; Huaguo Feng performed animal model constructions, performed RT-qPCR and drafted the manuscript; Qingbo Pan and Yanqin Wang performed analysis and data acquisition; Wenfeng Zhang and Jianping Gong help to revise the manuscript. The authors read and approved the final manuscript.

\section{Funding}

Huaguo Feng received funding from the National Natural Science Foundation of Chongqing (csct2019jcyj-msxmX0620); Wenfeng Zhang received funding from the National Natural Science Foundation of China (81801963); Jianping Gong received funding from the National Natural 
Science Foundation of China (81971880).

\section{Availability of data and material}

Data were available on request.

\section{Ethics approval and consent to participate}

This study was an analysis of the third-party anonymized databases with preexisting IRB approval.

\section{Consent for publication}

Not applicable.

\section{Competing interests}

The authors declare that they have no competing interests

\section{References}

1. Singer M, Deutschman CS, Seymour CW, Shankar-Hari M, Annane D, Bauer M, et al. The Third International Consensus Definitions for Sepsis and Septic Shock (Sepsis-3). JAMA. 2016;315(8):801-10.

2. van der Poll T, van de Veerdonk FL, Scicluna BP, Netea MG. The immunopathology of sepsis and potential therapeutic targets. Nat Rev Immunol. 2017;17(7):407-20.

3. Perner A, Gordon AC, De Backer D, Dimopoulos G, Russell JA, Lipman J, et al. Sepsis: frontiers in diagnosis, resuscitation and antibiotic therapy. Intensive Care Med. 2016;42(12):1958-69.

4. Rudd KE, Johnson SC, Agesa KM, Shackelford KA, Tsoi D, Kievlan DR, et al. Global, regional, and national sepsis incidence and mortality, 1990-2017: analysis for the Global Burden of Disease Study. Lancet. 2020;395(10219):200-11.

5. Fleischmann C, Scherag A, Adhikari NK, Hartog CS, Tsaganos T, Schlattmann P, et al. Assessment of Global Incidence and Mortality of Hospital-treated Sepsis. Current Estimates and Limitations. Am J Respir Crit Care Med. 2016;193(3):259-72.

6. Seymour CW, Gesten F, Prescott HC, Friedrich ME, Iwashyna TJ, Phillips GS, et al. Time to Treatment and Mortality during Mandated Emergency Care for Sepsis. N Engl J Med. 2017;376(23):2235-44.

7. dos Santos CC, Gattas DJ, Tsoporis JN, Smeding L, Kabir G, Masoom H, et al. Sepsis-induced myocardial depression is associated with transcriptional changes in energy metabolism and contractile related genes: a physiological and gene expression-based approach. Crit Care Med. 2010;38(3):894-902.

8. Klaude M, Mori M, Tjäder I, Gustafsson T, Wernerman J, Rooyackers O. Protein metabolism and gene expression in skeletal muscle of critically ill patients with sepsis. Clin Sci (Lond). 2012;122(3):133-42.

9. Golucci A, Marson F, Ribeiro AF, Nogueira R. Lipid profile associated with the systemic inflammatory response syndrome and sepsis in critically ill patients. Nutrition. 
2018;55-56:7-14.

10. Green P, Theilla M, Singer P. Lipid metabolism in critical illness. Curr Opin Clin Nutr Metab Care. 2016;19(2):111-5.

11. Yang L, Xie M, Yang M, Yu Y, Zhu S, Hou W, et al. PKM2 regulates the Warburg effect and promotes HMGB1 release in sepsis. Nat Commun. 2014;5:4436.

12. Xie M, Yu Y, Kang R, Zhu S, Yang L, Zeng L, et al. PKM2-dependent glycolysis promotes NLRP3 and AIM2 inflammasome activation. Nat Commun. 2016;7:13280.

13. Paciullo F, Fallarino F, Bianconi V, Mannarino MR, Sahebkar A, Pirro M. PCSK9 at the crossroad of cholesterol metabolism and immune function during infections. J Cell Physiol. 2017;232(9):2330-8.

14. Boyd JH, Fjell CD, Russell JA, Sirounis D, Cirstea MS, Walley KR. Increased Plasma PCSK9 Levels Are Associated with Reduced Endotoxin Clearance and the Development of Acute Organ Failures during Sepsis. J Innate Immun. 2016;8(2):211-20.

15. Walley KR. Role of lipoproteins and proprotein convertase subtilisin/kexin type 9 in endotoxin clearance in sepsis. Curr Opin Crit Care. 2016;22(5):464-9.

16. Jiang P, Gu S, Pan D, Fu J, Sahu A, Hu X, et al. Signatures of T cell dysfunction and exclusion predict cancer immunotherapy response. Nat Med. 2018;24(10):1550-8.

17. Patil NK, Bohannon JK, Sherwood ER. Immunotherapy: A promising approach to reverse sepsis-induced immunosuppression. Pharmacol Res. 2016;111:688-702.

18. Taccone FS, Su F, De Deyne C, Abdellhai A, Pierrakos C, He X, et al. Sepsis is associated with altered cerebral microcirculation and tissue hypoxia in experimental peritonitis. Crit Care Med. 2014;42(2):e114-22.

19. Van Wyngene L, Vandewalle J, Libert C. Reprogramming of basic metabolic pathways in microbial sepsis: therapeutic targets at last. EMBO Mol Med. 2018;10(8).

20. Belaaouaj A, Kim KS, Shapiro SD. Degradation of outer membrane protein A in Escherichia coli killing by neutrophil elastase. Science. 2000;289(5482):1185-8.

21. Jundi B, Ryu H, Lee DH, Abdulnour RE, Engstrom BD, Duvall MG, et al. Leukocyte function assessed via serial microlitre sampling of peripheral blood from sepsis patients correlates with disease severity. Nat Biomed Eng. 2019;3(12):961-73.

22. Zhang S, Li N, Chen W, Fu Q, Liu Y. Time Series Gene Expression Profiles Analysis Identified Several Potential Biomarkers for Sepsis. DNA Cell Biol. 2020;39(10):1862-71.

23. Brightbill HD, Libraty DH, Krutzik SR, Yang RB, Belisle JT, Bleharski JR, et al. Host defense mechanisms triggered by microbial lipoproteins through toll-like receptors. Science. 1999;285(5428):732-6.

24. Aliprantis AO, Yang RB, Mark MR, Suggett S, Devaux B, Radolf JD, et al. Cell activation and apoptosis by bacterial lipoproteins through toll-like receptor-2. Science. 1999;285(5428):736-9.

25. Lancioni CL, Li Q, Thomas JJ, Ding X, Thiel B, Drage MG, et al. Mycobacterium tuberculosis lipoproteins directly regulate human memory $\mathrm{CD} 4(+) \mathrm{T}$ cell activation via Toll-like receptors 1 and 2. Infect Immun. 2011;79(2):663-73.

26. Wang L, Li Y, Wang X, Wang P, Essandoh K, Cui S, et al. GDF3 Protects Mice against Sepsis-Induced Cardiac Dysfunction and Mortality by Suppression of Macrophage Pro-Inflammatory Phenotype. Cells. 2020;9(1).

27. Yang Q, Cao K, Jin G, Zhang J. Hsa-miR-346 plays a role in the development of sepsis by 
downregulating SMAD3 expression and is negatively regulated by lncRNA MALAT1. Mol Cell Probes. 2019;47:101444.

28. Williams B, Neder J, Cui P, Suen A, Tanaka K, Zou L, et al. Toll-like receptors 2 and 7 mediate coagulation activation and coagulopathy in murine sepsis. J Thromb Haemost. 2019;17(10):1683-93.

29. Gil D, Gutiérrez D, Alarcón B. Intracellular redistribution of nucleolin upon interaction with the CD3epsilon chain of the $\mathrm{T}$ cell receptor complex. $\mathrm{J}$ Biol Chem. 2001;276(14):11174-9.

30. Almansa R, Heredia-Rodríguez M, Gomez-Sanchez E, Andaluz-Ojeda D, Iglesias V, Rico $\mathrm{L}$, et al. Transcriptomic correlates of organ failure extent in sepsis. J Infect. 2015;70(5):445-56.

31. Choi DJ, An J, Jou I, Park SM, Joe EH. A Parkinson's disease gene, DJ-1, regulates anti-inflammatory roles of astrocytes through prostaglandin $\mathrm{D}(2)$ synthase expression. Neurobiol Dis. 2019;127:482-91.

32. Zhao W, Jiang B, Hu H, Zhang S, Lv S, Yuan J, et al. Lack of CUL4B leads to increased abundance of GFAP-positive cells that is mediated by PTGDS in mouse brain. Hum Mol Genet. 2015;24(16):4686-97.

33. Yu JY, Zhang B, Peng L, Wu CH, Cao H, Zhong JF, et al. Repositioning of Memantine as a Potential Novel Therapeutic Agent against Meningitic E. coli-Induced Pathogenicities through Disease-Associated Alpha7 Cholinergic Pathway and RNA Sequencing-Based Transcriptome Analysis of Host Inflammatory Responses. PLoS One. 2015;10(5):e0121911.

34. Jamieson D, Cresti N, Bray J, Sludden J, Griffin MJ, Hawsawi NM, et al. Two minor NQO1 and NQO2 alleles predict poor response of breast cancer patients to adjuvant doxorubicin and cyclophosphamide therapy. Pharmacogenet Genomics. 2011;21(12):808-19.

35. Yu KD, Di GH, Yuan WT, Fan L, Wu J, Hu Z, et al. Functional polymorphisms, altered gene expression and genetic association link NRH:quinone oxidoreductase 2 to breast cancer with wild-type p53. Hum Mol Genet. 2009;18(13):2502-17.

36. Hubackova M, Vaclavikova R, Ehrlichova M, Mrhalova M, Kodet R, Kubackova K, et al. Association of superoxide dismutases and $\mathrm{NAD}(\mathrm{P}) \mathrm{H}$ quinone oxidoreductases with prognosis of patients with breast carcinomas. Int J Cancer. 2012;130(2):338-48.

37. Das A, Kole L, Wang L, Barrios R, Moorthy B, Jaiswal AK. BALT development and augmentation of hyperoxic lung injury in mice deficient in NQO1 and NQO2. Free Radic Biol Med. 2006;40(10):1843-56.

38. Otto GP, Sossdorf M, Claus RA, Rödel J, Menge K, Reinhart K, et al. The late phase of sepsis is characterized by an increased microbiological burden and death rate. Crit Care. 2011;15(4):R183.

39. Torgersen C, Moser P, Luckner G, Mayr V, Jochberger S, Hasibeder WR, et al. Macroscopic postmortem findings in 235 surgical intensive care patients with sepsis. Anesth Analg. 2009;108(6):1841-7.

40. Jensen IJ, Sjaastad FV, Griffith TS, Badovinac VP. Sepsis-Induced T Cell Immunoparalysis: The Ins and Outs of Impaired T Cell Immunity. J Immunol. 2018;200(5):1543-53.

41. Krautz C, Maier SL, Brunner M, Langheinrich M, Giamarellos-Bourboulis EJ, Gogos C, et 
al. Reduced circulating B cells and plasma IgM levels are associated with decreased survival in sepsis - A meta-analysis. J Crit Care. 2018;45:71-5.

42. Suzuki K, Inoue S, Kametani Y, Komori Y, Chiba S, Sato T, et al. Reduced Immunocompetent B Cells and Increased Secondary Infection in Elderly Patients With Severe Sepsis. Shock. 2016;46(3):270-8.

43. Feng T, Liao X, Yang X, Yang C, Lin F, Guo Y, et al. A shift toward inhibitory receptors and impaired effector functions on NK cells contribute to immunosuppression during sepsis. J Leukoc Biol. 2020;107(1):57-67.

44. Shen XF, Cao K, Jiang JP, Guan WX, Du JF. Neutrophil dysregulation during sepsis: an overview and update. J Cell Mol Med. 2017;21(9):1687-97.

45. Luo L, Xu M, Liao D, Deng J, Mei H, Hu Y. PECAM-1 protects against DIC by dampening inflammatory responses via inhibiting macrophage pyroptosis and restoring vascular barrier integrity. Transl Res. 2020;222:1-16.

46. Delabranche X, Quenot JP, Lavigne T, Mercier E, François B, Severac F, et al. Early Detection of Disseminated Intravascular Coagulation During Septic Shock: A Multicenter Prospective Study. Crit Care Med. 2016;44(10):e930-9.

47. Tapia P, Gatica S, Cortés-Rivera C, Otero C, Becerra A, Riedel CA, et al. Circulating Endothelial Cells From Septic Shock Patients Convert to Fibroblasts Are Associated With the Resuscitation Fluid Dose and Are Biomarkers for Survival Prediction. Crit Care Med. 2019;47(7):942-50.

48. Patil NK, Guo Y, Luan L, Sherwood ER. Targeting Immune Cell Checkpoints during Sepsis. Int J Mol Sci. 2017;18(11).

49. Venet F, Monneret G. Advances in the understanding and treatment of sepsis-induced immunosuppression. Nat Rev Nephrol. 2018;14(2):121-37.

50. Huang X, Venet F, Wang YL, Lepape A, Yuan Z, Chen Y, et al. PD-1 expression by macrophages plays a pathologic role in altering microbial clearance and the innate inflammatory response to sepsis. Proc Natl Acad Sci U S A. 2009;106(15):6303-8.

51. Zhang Y, Zhou Y, Lou J, Li J, Bo L, Zhu K, et al. PD-L1 blockade improves survival in experimental sepsis by inhibiting lymphocyte apoptosis and reversing monocyte dysfunction. Crit Care. 2010;14(6):R220.

52. Shindo Y, Unsinger J, Burnham CA, Green JM, Hotchkiss RS. Interleukin-7 and anti-programmed cell death 1 antibody have differing effects to reverse sepsis-induced immunosuppression. Shock. 2015;43(4):334-43.

53. Chang KC, Burnham CA, Compton SM, Rasche DP, Mazuski RJ, McDonough JS, et al. Blockade of the negative co-stimulatory molecules PD-1 and CTLA-4 improves survival in primary and secondary fungal sepsis. Crit Care. 2013;17(3):R85.

54. Huang X, Chen Y, Chung CS, Yuan Z, Monaghan SF, Wang F, et al. Identification of B7-H1 as a novel mediator of the innate immune/proinflammatory response as well as a possible myeloid cell prognostic biomarker in sepsis. J Immunol. 2014;192(3):1091-9.

55. Hotchkiss RS, Colston E, Yende S, Crouser ED, Martin GS, Albertson T, et al. Immune checkpoint inhibition in sepsis: a Phase $1 \mathrm{~b}$ randomized study to evaluate the safety, tolerability, pharmacokinetics, and pharmacodynamics of nivolumab. Intensive Care Med. 2019;45(10):1360-71.

56. Hotchkiss RS, Colston E, Yende S, Angus DC, Moldawer LL, Crouser ED, et al. Immune 
Checkpoint Inhibition in Sepsis: A Phase 1b Randomized, Placebo-Controlled, Single Ascending Dose Study of Antiprogrammed Cell Death-Ligand 1 Antibody (BMS-936559). Crit Care Med. 2019;47(5):632-42.

57. Wang J, Sanmamed MF, Datar I, Su TT, Ji L, Sun J, et al. Fibrinogen-like Protein 1 Is a Major Immune Inhibitory Ligand of LAG-3. Cell. 2019;176(1-2):334-47.e12.

58. Niu B, Zhou F, Su Y, Wang L, Xu Y, Yi Z, et al. Different Expression Characteristics of LAG3 and PD-1 in Sepsis and Their Synergistic Effect on T Cell Exhaustion: A New Strategy for Immune Checkpoint Blockade. Front Immunol. 2019;10:1888.

59. Wang Q, Li M, Yang M, Yang Y, Song F, Zhang W, et al. Analysis of immune-related signatures of lung adenocarcinoma identified two distinct subtypes: implications for immune checkpoint blockade therapy. Aging (Albany NY). 2020;12(4):3312-39.

60. Cao R, Yuan L, Ma B, Wang G, Tian Y. Tumour microenvironment (TME) characterization identified prognosis and immunotherapy response in muscle-invasive bladder cancer (MIBC). Cancer Immunol Immunother. 2020.

61. Ren Q, Zhu P, Zhang H, Ye T, Liu D, Gong Z, et al. Identification and validation of stromal-tumor microenvironment-based subtypes tightly associated with PD-1/PD-L1 immunotherapy and outcomes in patients with gastric cancer. Cancer Cell Int. 2020;20:92.

62. Cao R, Yuan L, Ma B, Wang G, Tian Y. Immune-related long non-coding RNA signature identified prognosis and immunotherapeutic efficiency in bladder cancer (BLCA). Cancer Cell Int. 2020;20:276.

A

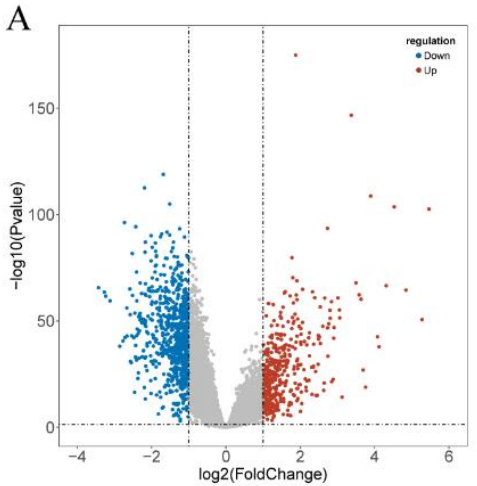

B

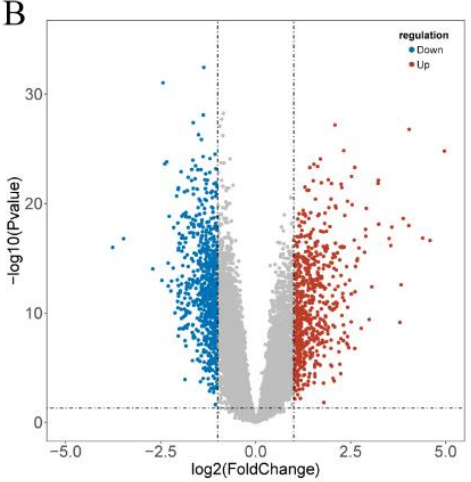

$\mathrm{C}_{\text {Metabolism-related gene }}$

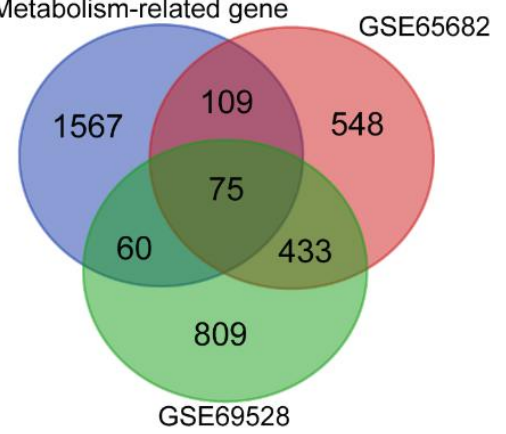

Figure1. Identification of DEMAGs in GSE65682 and GSE69528 datasets. (A) Volcano plot for DEGs in the GSE65682 dataset (B) Volcano plot for DEGs in the GSE69528 dataset (Red 
points represented up-regulated genes, while blue points represented down-regulated genes, Grey points represented genes with no significant difference) (C)Venn diagram for overlapping genes between DEGs in two datasets and MAGs.

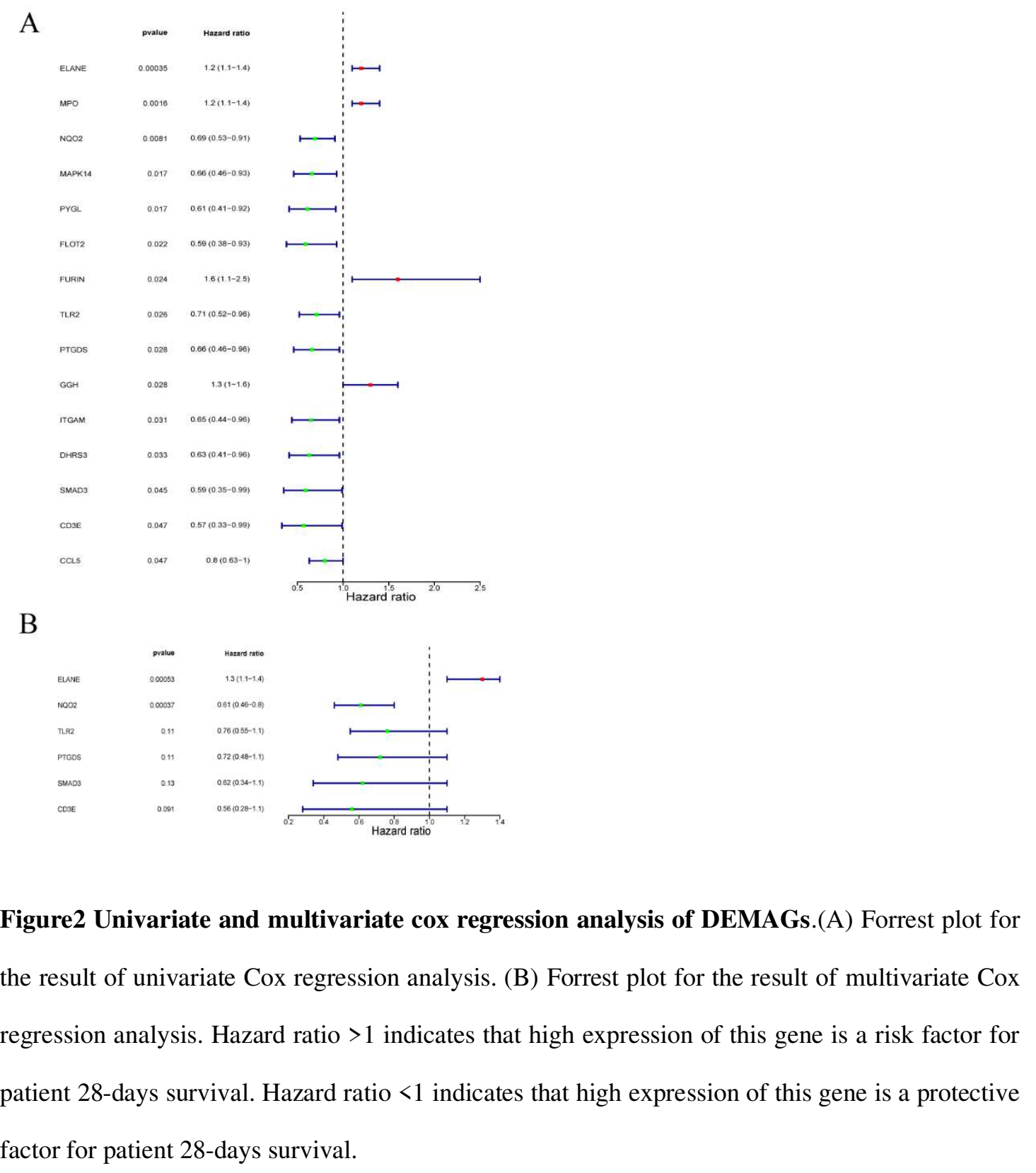


A

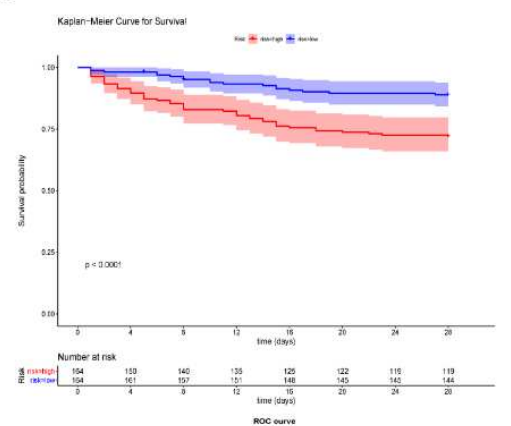

B

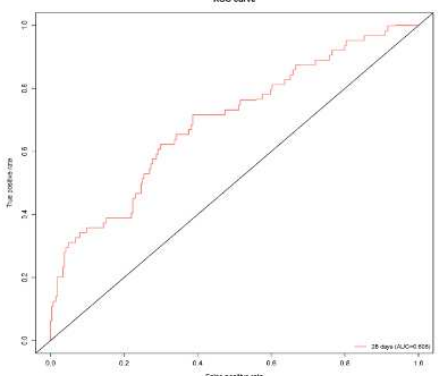

C

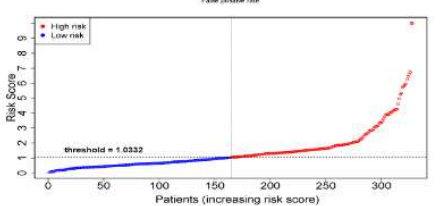

$\mathrm{D}$

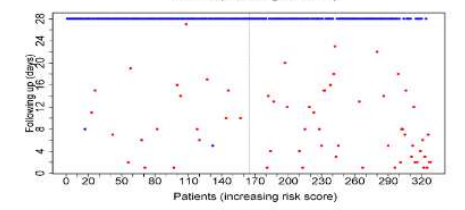

E

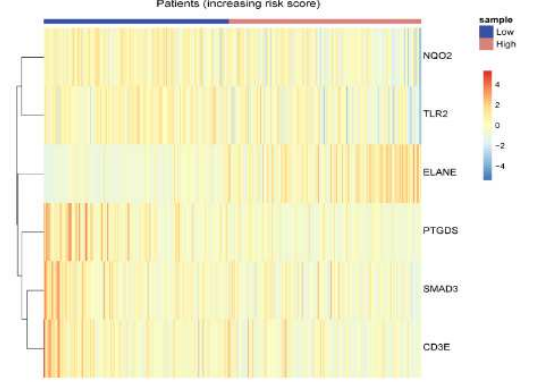

$\mathrm{F}$
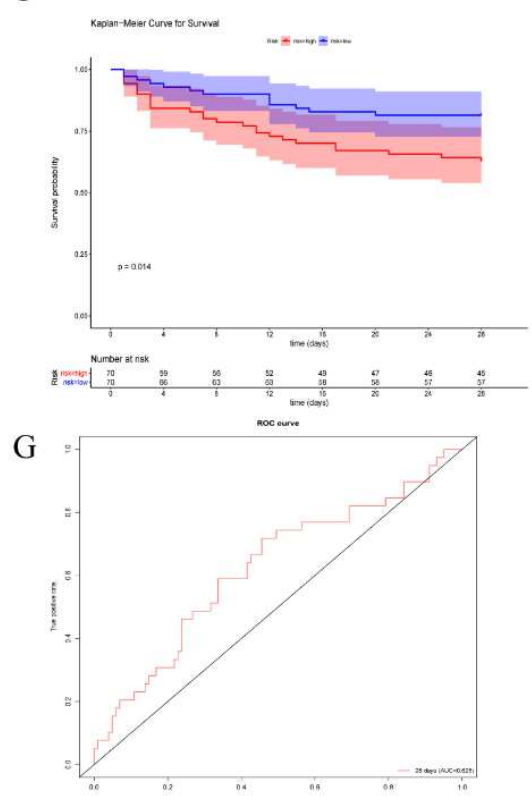

$\mathrm{H}$

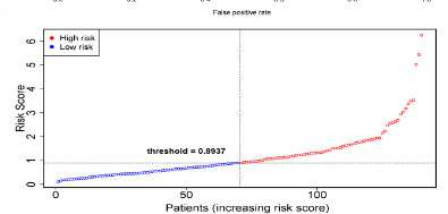

I

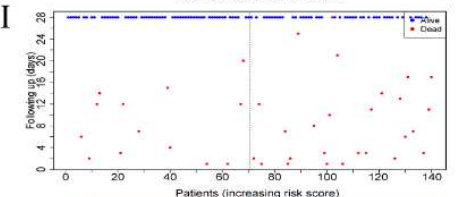

$\mathrm{J}$

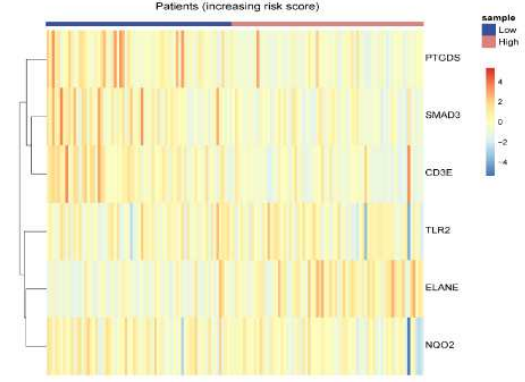

Figure3 Performance evaluation of the six-gene signature for survival prediction in the training and validation set. (A), (F) Kaplan-Meier curve of the six-gene signature (B), (G) Time-dependent ROC curve of the six-gene signature. (C), (H) The risk score distribution of patients. (D), (I) The 28-days survival situation of patients. (E), (J) The heatmap of the six genes expression profiles. (A E) Training set $(\mathrm{F} \sim \mathrm{J})$ Validation set 
A

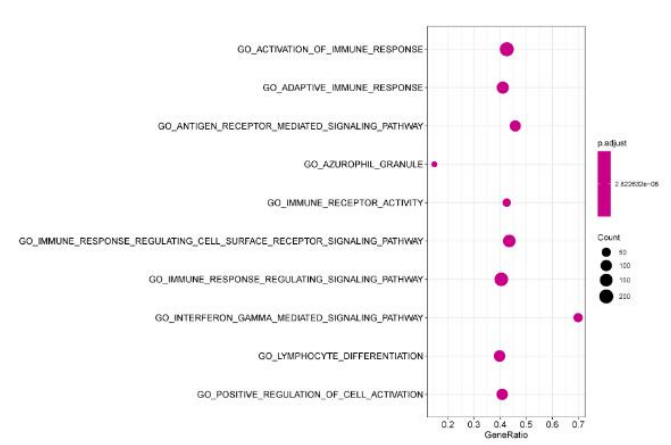

C

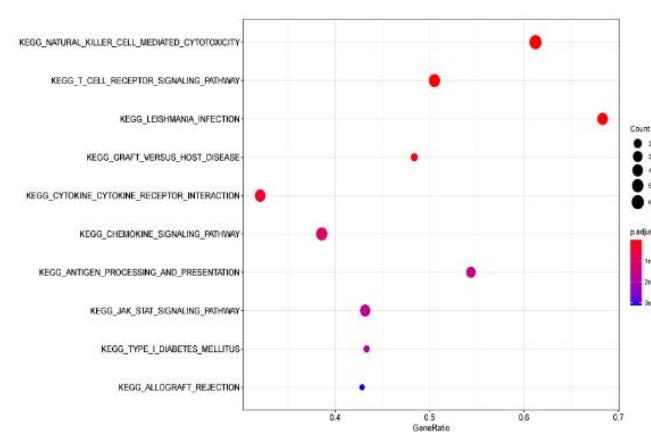

B

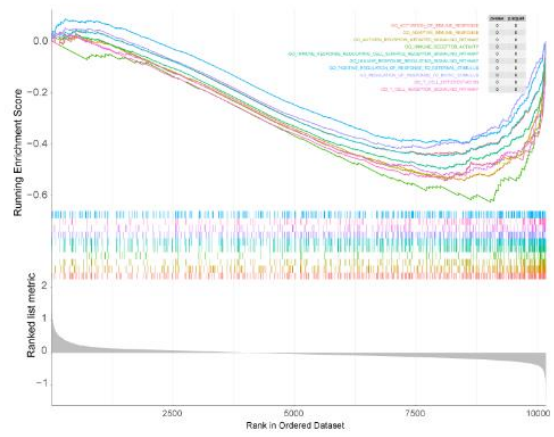

D

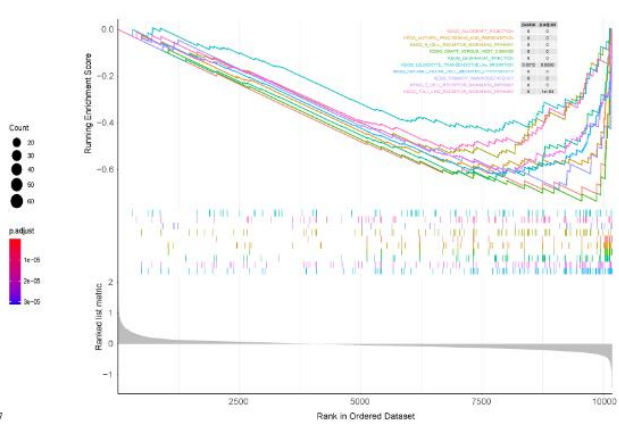

Figure4 Gene set enrichment analysis based on the six-gene signature. (A B) GSEA revealed the significantly enriched GO gene sets (Top 10). (C D) GSEA revealed the significantly enriched KEGG gene sets (Top 10). 
A

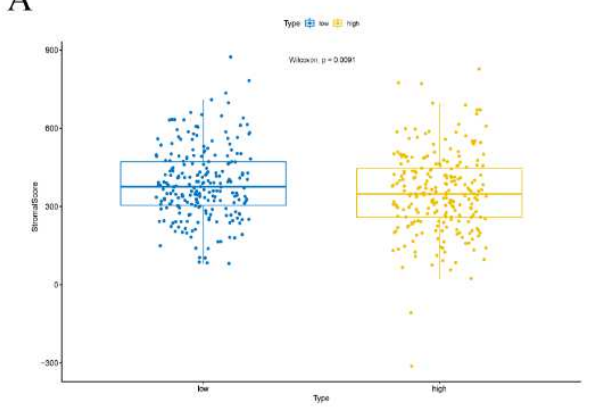

$\mathrm{C}$

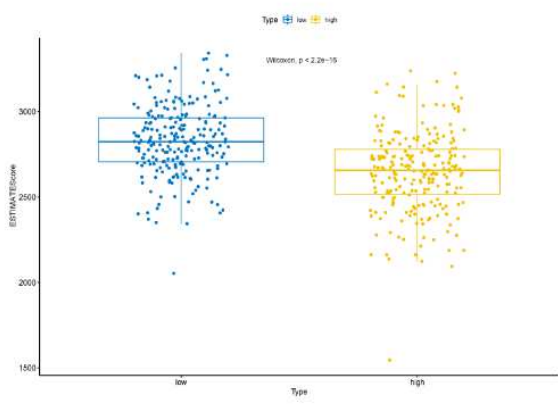

$\mathrm{E}$

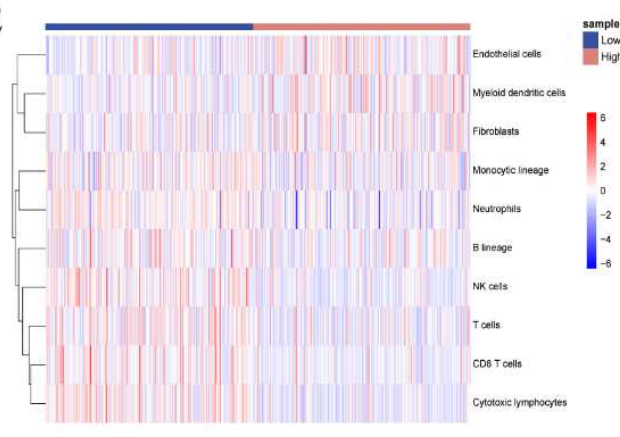

B

D
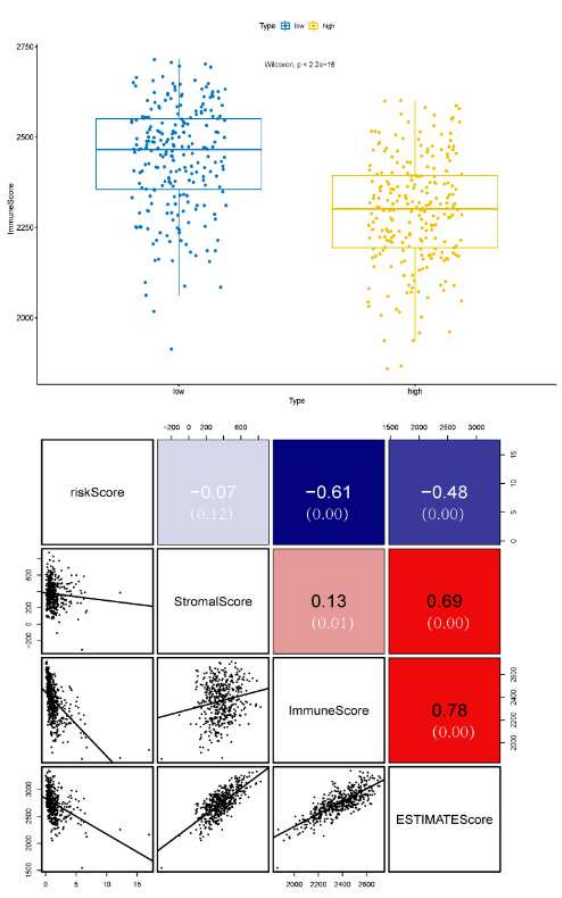

Type 由 bion it iox

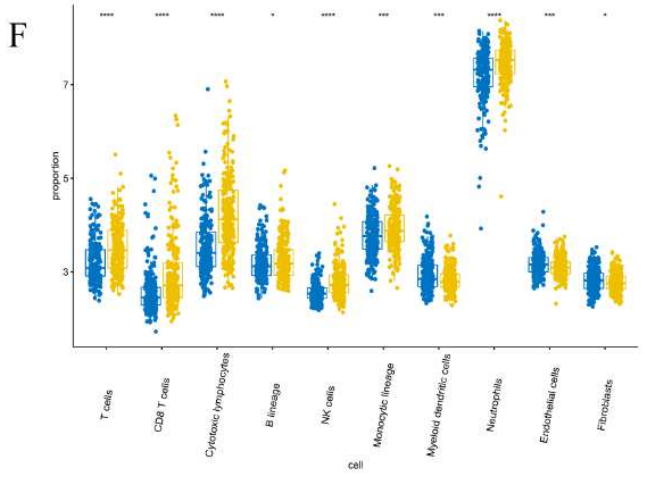

Figure5 Immune microenvironment of high and low risk groups. (A)Boxplot of stromal score in high- and low-risk groups $(\mathrm{P}<0.05)$. (B)Boxplot of immune score in high- and low-risk groups $(\mathrm{P}<0.05)$. (C) Boxplot of ESTIMATES score in high- and low-risk groups $(\mathrm{P}<0.05)$. (D) Scatterplot and heatmap of correlation between risk score and stromal score, immune score and ESTIMATES score. (E)Heatmap of immune cells calculated by MCP-counter. (F) Boxplot of immune cells in high- and low-risk group. $*$ represents $\mathrm{p}<0.05$, ** represents $\mathrm{p}<0.01, * * *$ represents $\mathrm{p}<0.001, \quad * * * *$ represents $\mathrm{p}<0.0001$ 
A

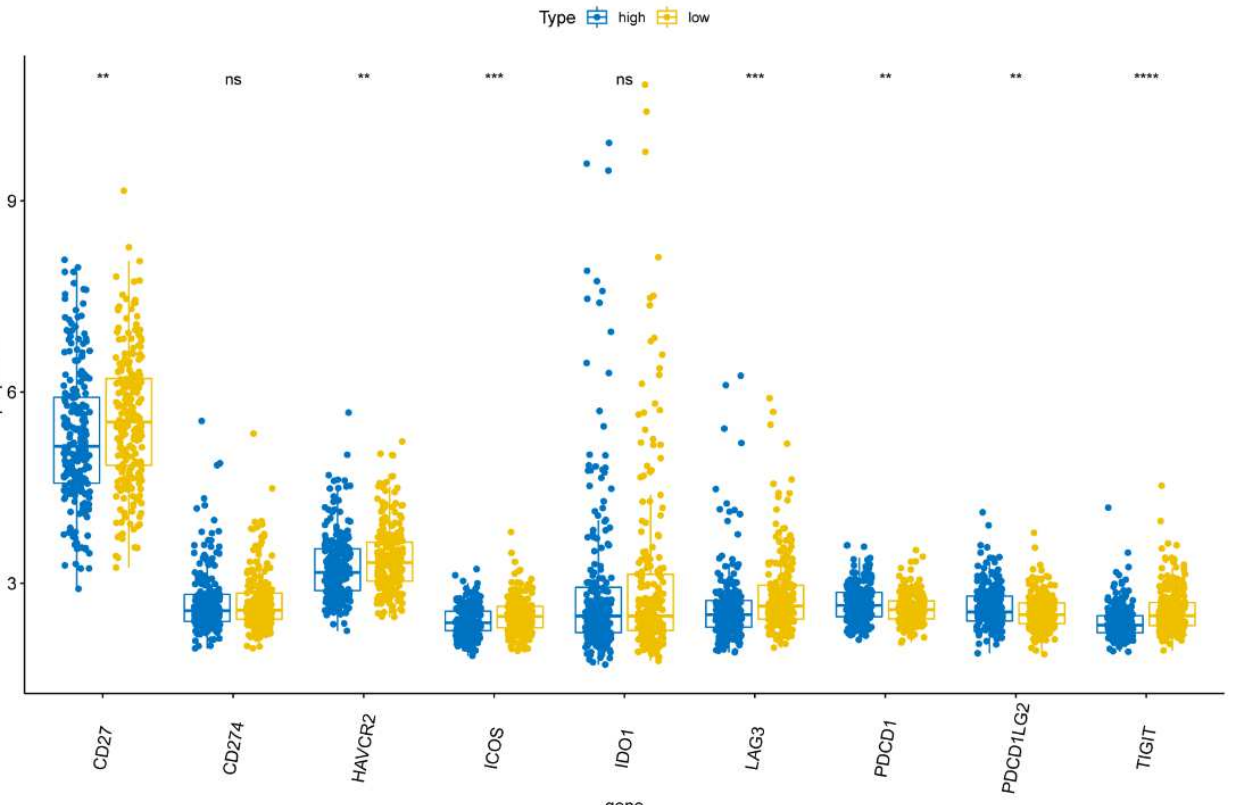

B

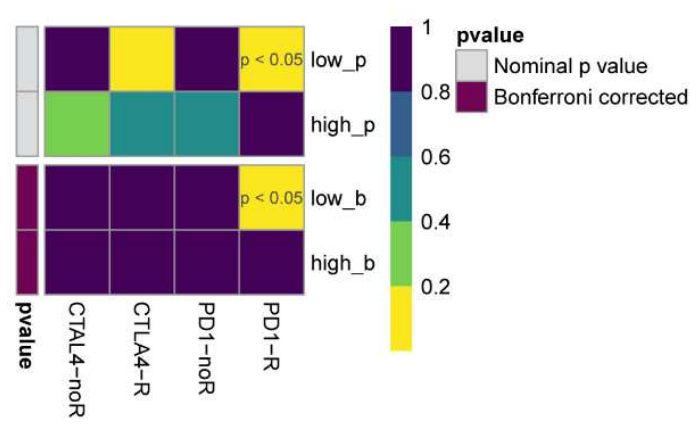

$\mathrm{C}$

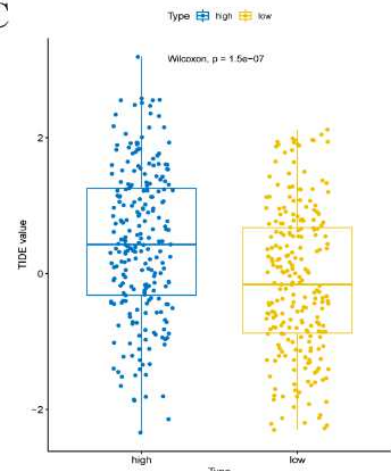

Figure6 Expression of immune checkpoint molecule and immunotherapy response of the high- and low-risk groups. (A) Boxplot of immune checkpoint molecules in high- and low-risk groups. ns represents no significance, * represents $\mathrm{P}<0.05$, ** represents $\mathrm{P}<0.01$, *** represents $\mathrm{P}<0.001, \quad * * * *$ represents $\mathrm{P}<0.0001$. (B) Submap algorithm manifested that low-risk group could be more sensitive to the programmed cell death protein 1 (PD-1) inhibitor (Bonferroni-corrected P $<0.05)$.(C) Boxplot of TIDE score in high- and low-risk groups. The Boxplot showed that the TIDE score of low-risk group was lower (Wilcoxon $\mathrm{P}=1.5 \mathrm{e}-07$ ), indicating that the low-risk group was more sensitive to immunotherapy. 
A

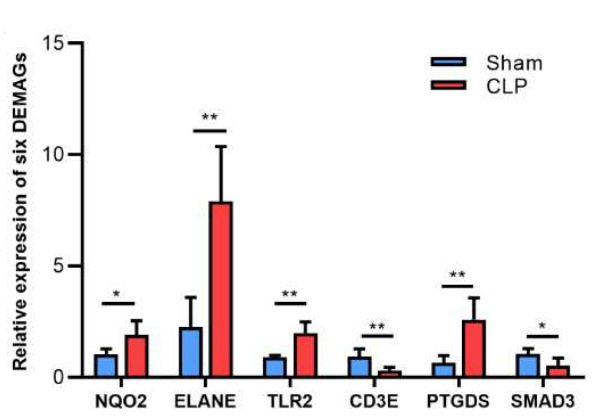

C

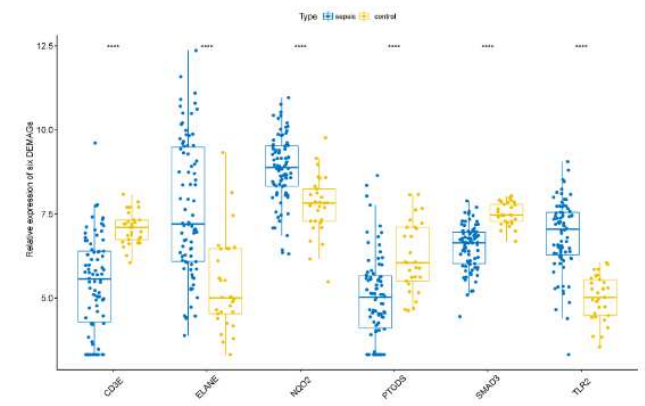

B

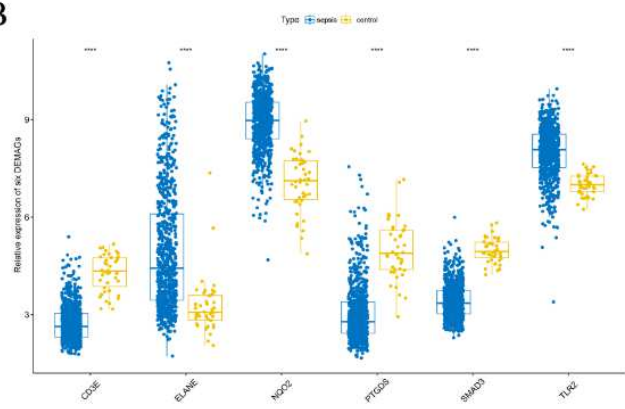

Figure7 Expression of six DEMAGs in sepsis mice model and two datasets. (A) Expression levels of DEMAGs in GSE65682 in mice model determined by quantitative real-time-PCR. Sham: The control group mice constructed by sham operation n=5; CLP: Sepsis mice model contrasted by cecal ligation and puncture $n=5$. (B) Expression levels of DEMAGs in GSE65682. (C) Expression levels of DEMAGs in GSE69528. Student's t-test * represents $\mathrm{p}<0.05$, ** represents $\mathrm{p}<0.01, * * *$ represents $\mathrm{P}<0.001, * * * *$ represents $\mathrm{P}<0.0001$. DEMAGs: Differentially expressed metabolism-associated genes. 


\section{Figures}
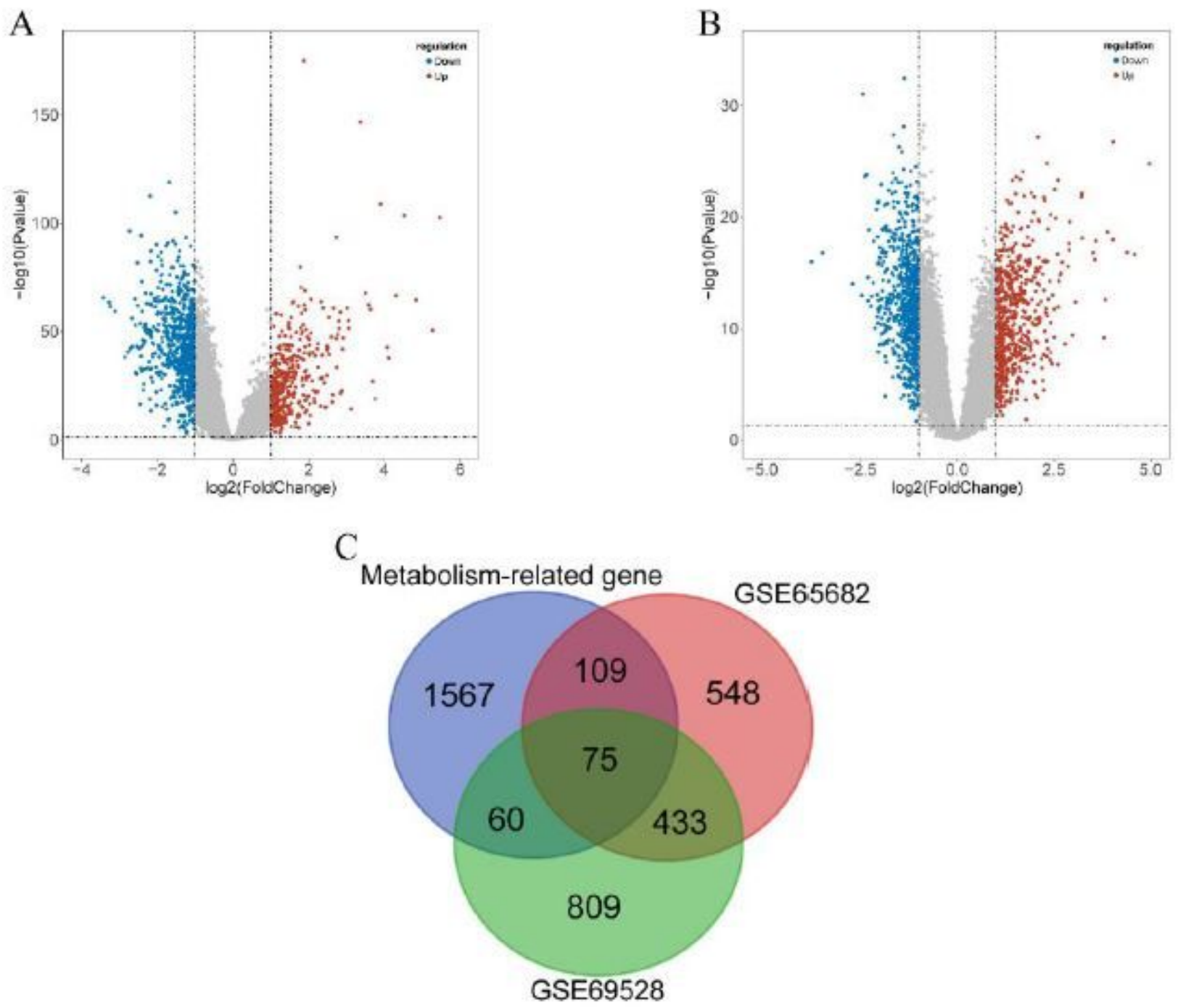

\section{Figure 1}

Identification of DEMAGs in GSE65682 and GSE69528 datasets. (Volcano plot for DEGs in the GSE65682 d ataset (B) Volcano plot for DEGs in the GSE69528 dataset (Red points represented up points represented up-regulated genes, while blue points regulated genes, while blue points represented downrepresented down-regulatedregulated genesgenes, , Grey Grey points represented genes with no significant difference) (C)Venn diagpoints represented genes with no significant difference) (C)Venn diagram for overlapping genes ram for overlapping genes betbetween ween DEGsDEGs in in two datasets andtwo datasets and MAGsMAGs.. 
A

\begin{tabular}{|c|c|c|}
\hline & pualue & Hezard ratio \\
\hline ELARE & 0.00035 & $1.2(t: 1-14)$ \\
\hline MPO & 00010 & $1.2(1.1-1.4)$ \\
\hline $\mathrm{NGO2}$ & 00081 & $0.09(0.53-0.91)$ \\
\hline MAPK 14 & 0.017 & $0.60(0.46-0.83)$ \\
\hline PYG. & 0.017 & $0.61(0.41-0.82)$ \\
\hline FLOT2 & 0.022 & $0.50(0.36-0.93)$ \\
\hline FURIN & 0.024 & $16\langle 1.1-2.5\rangle$ \\
\hline TLR2 & 0.028 & $0.71(0.52-0.86)$ \\
\hline PTODS & 0.028 & $0.66(0.46-0.96)$ \\
\hline GGH & 0.028 & $1.3(1-1.6)$ \\
\hline ITEAM & 0.031 & $D .65(0.44=0.86)$ \\
\hline DHRS3 & 0.033 & $0.83\{0.41-0.96\}$ \\
\hline SMAD3 & ooes & $0.59(0.35-0.99)$ \\
\hline Cose & 0047 & $0.57(0.35-0.99)$ \\
\hline cas & 0.047 & $08(063-1)$ \\
\hline
\end{tabular}

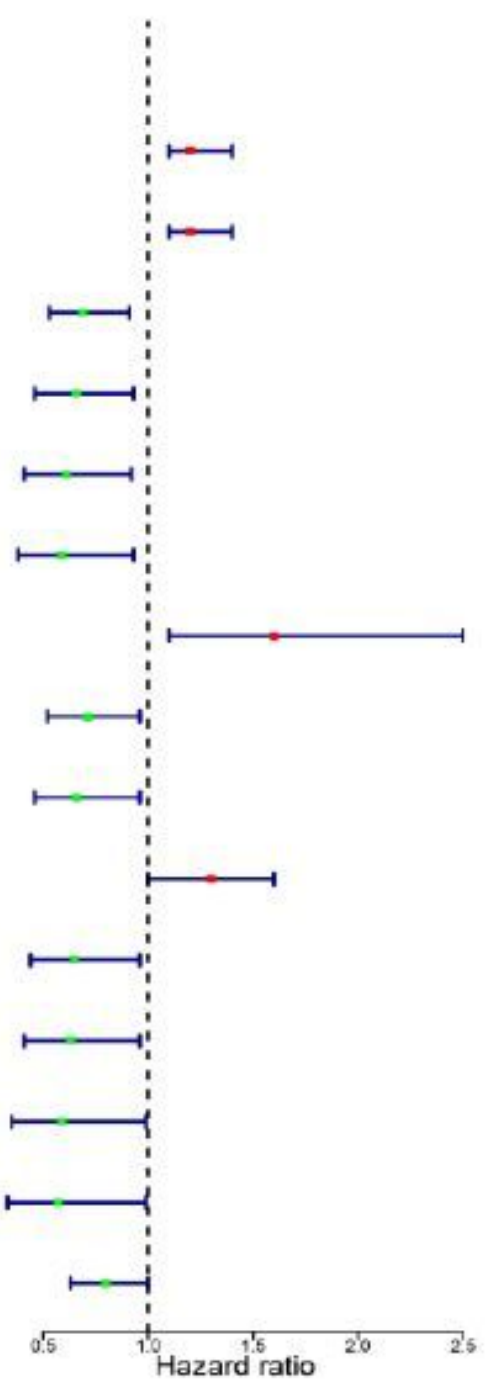

B

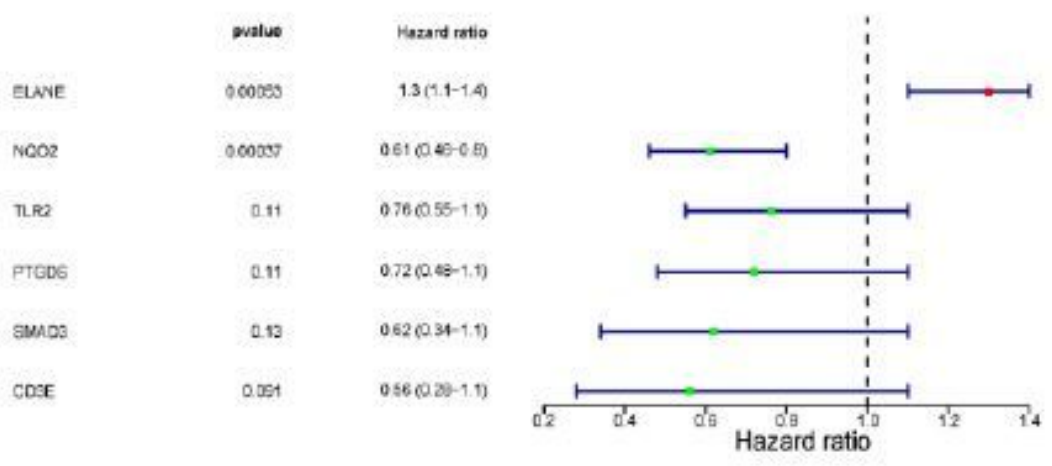

\section{Figure 2}

Univariate and multivariate cox regression analysis of DEMAGs ... Forrest plot for the result of univariate Cox regression analysis. (B) Forrest plot for the result of multivariate Cox regression analysis. Hazard ratio $>1$ indicates that high expression of this gene is a risk factor for patient 28 days survival. Hazard ratio $<1$ indicates that high expression of this gene is a protective factor for patient 28 days survival. 
A

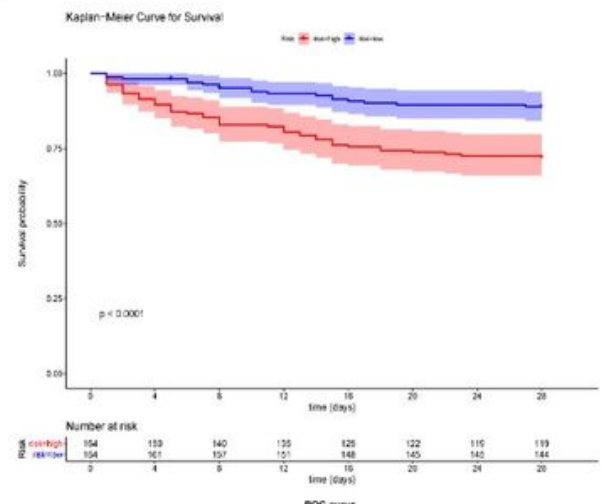

B

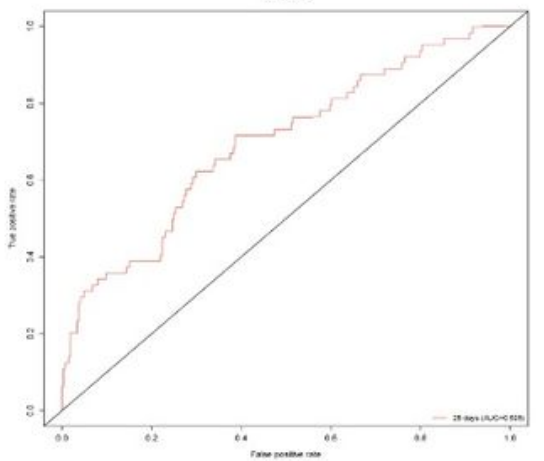

C

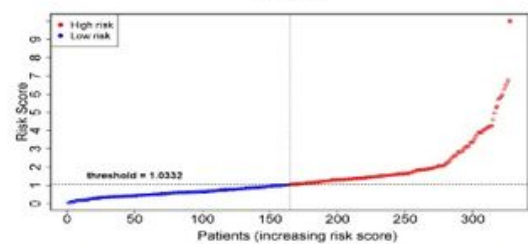

D

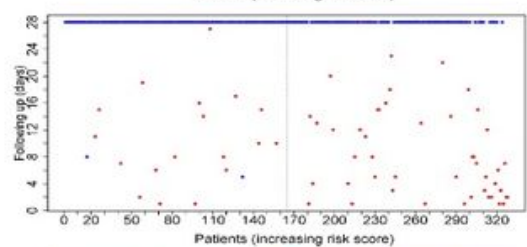

E

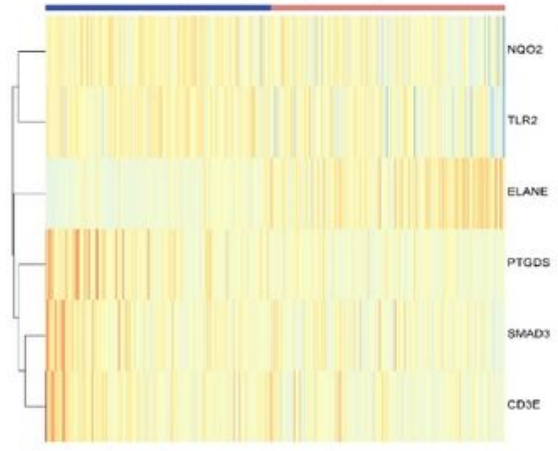

F

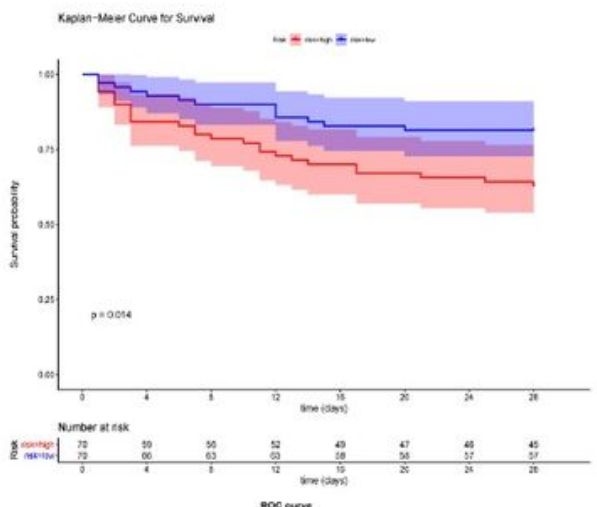

G

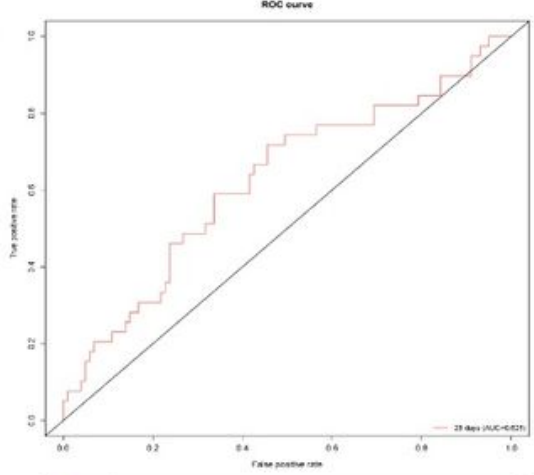

H
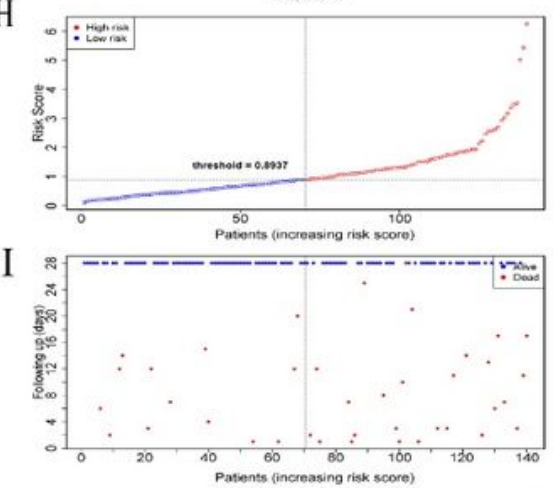

J

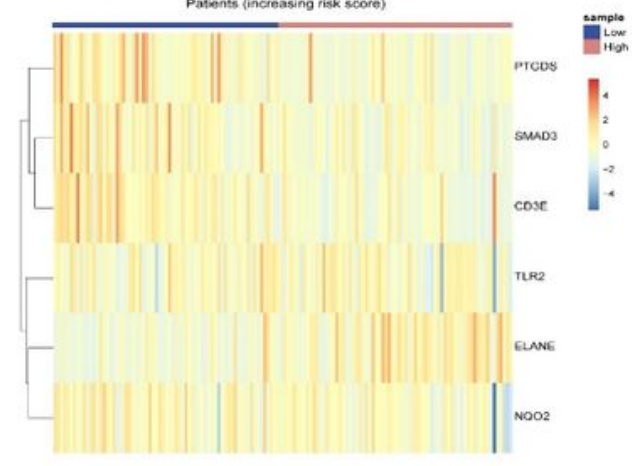

\section{Figure 3}

Performance evaluation of the six gene signature for survival prediction in the training and validation set. (A ), (F) Kaplan Meier curve of the six gene signature (B ), (G Time dependent ROC curve of the six gene signature. (C ), (H The risk score distribution of patients. (D ), (I T he 28 days survival situation of patients . (E ), (J ) The heatmap of the six genes expression profiles. (A E) Training se $t(F \sim J)$ Validation set 
A

B
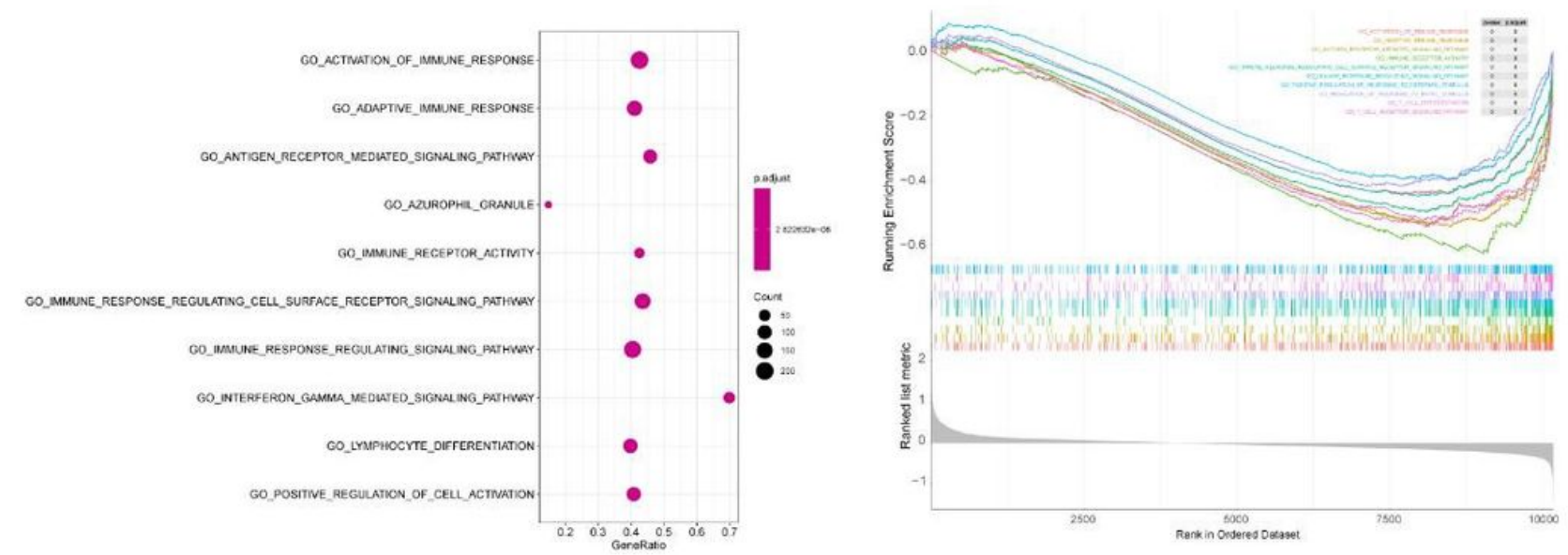

$\mathrm{C}$

$\mathrm{D}$
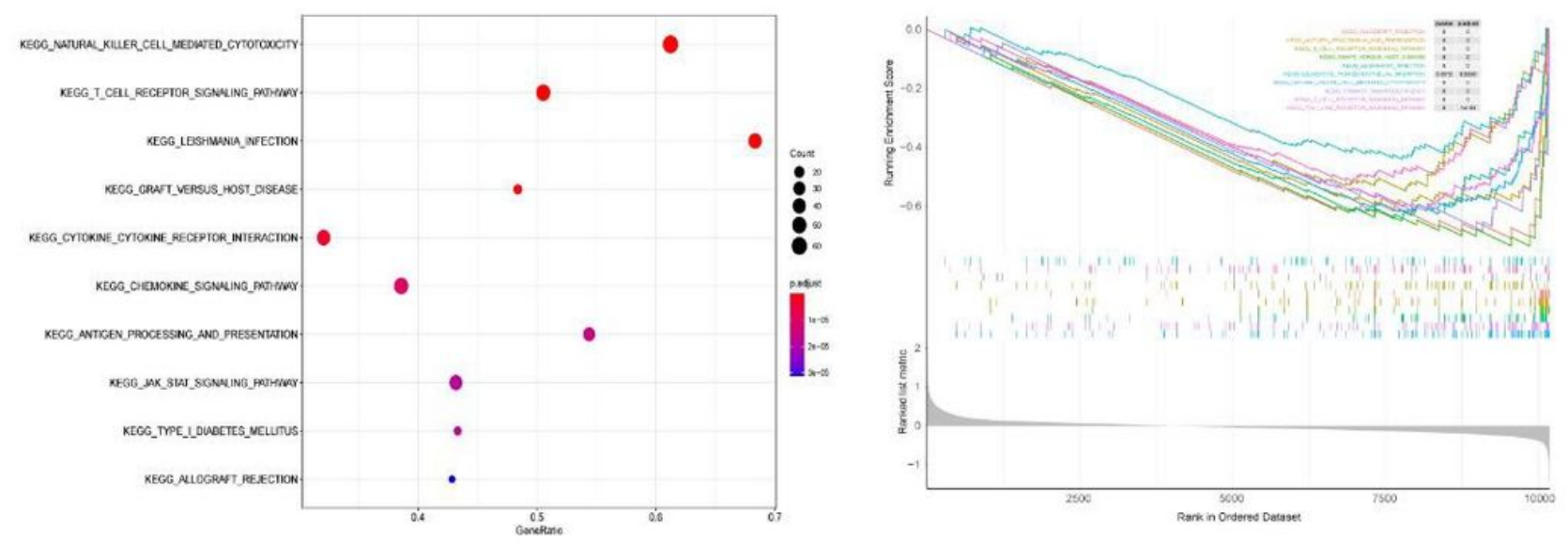

Figure 4

Gene set enrichment analysis based on the six gene signature. (A B) GSEA revealed the significantly enriched GO gene sets (Top 10). (C D) GSEA revealed the significantly enriched KEGG gene sets (Top 10). 
A

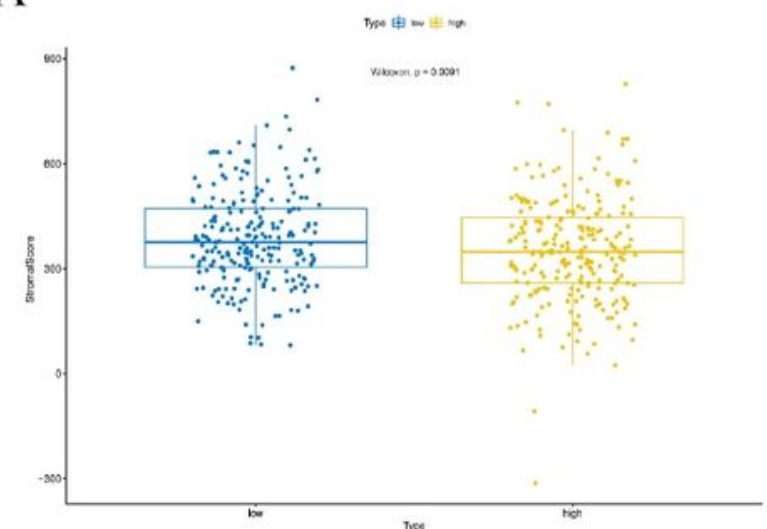

$\mathrm{C}$

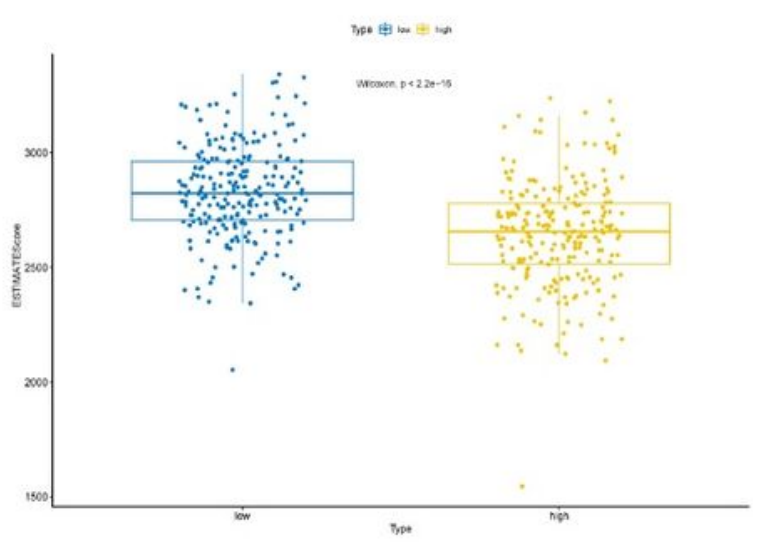

$\mathrm{E}$

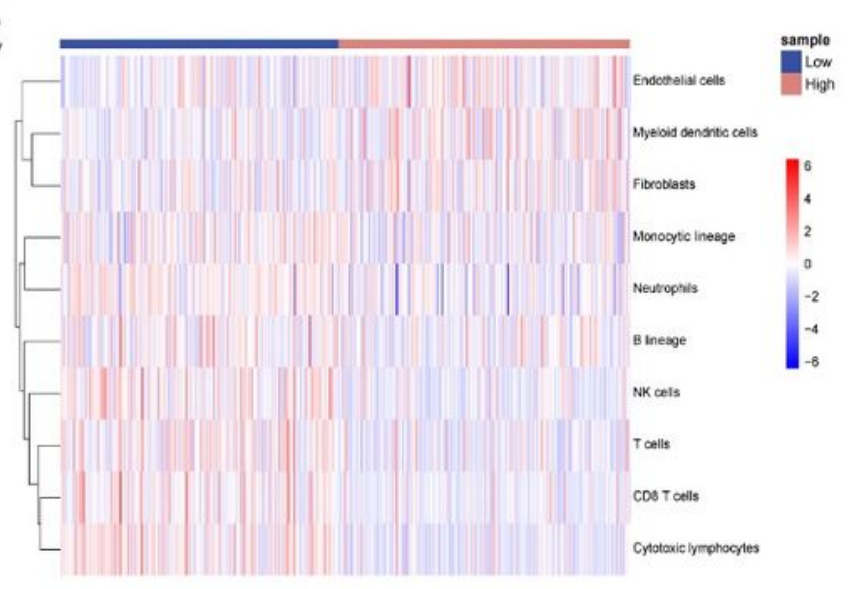

$\mathrm{B}$

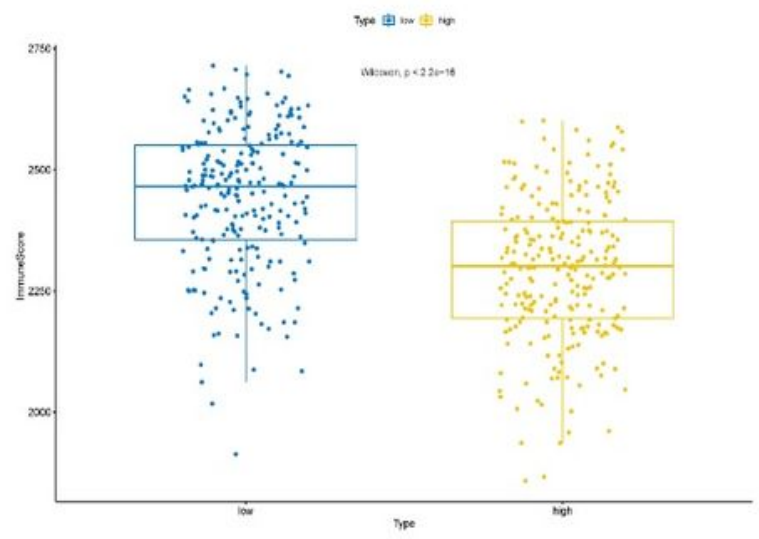

$\mathrm{D}$
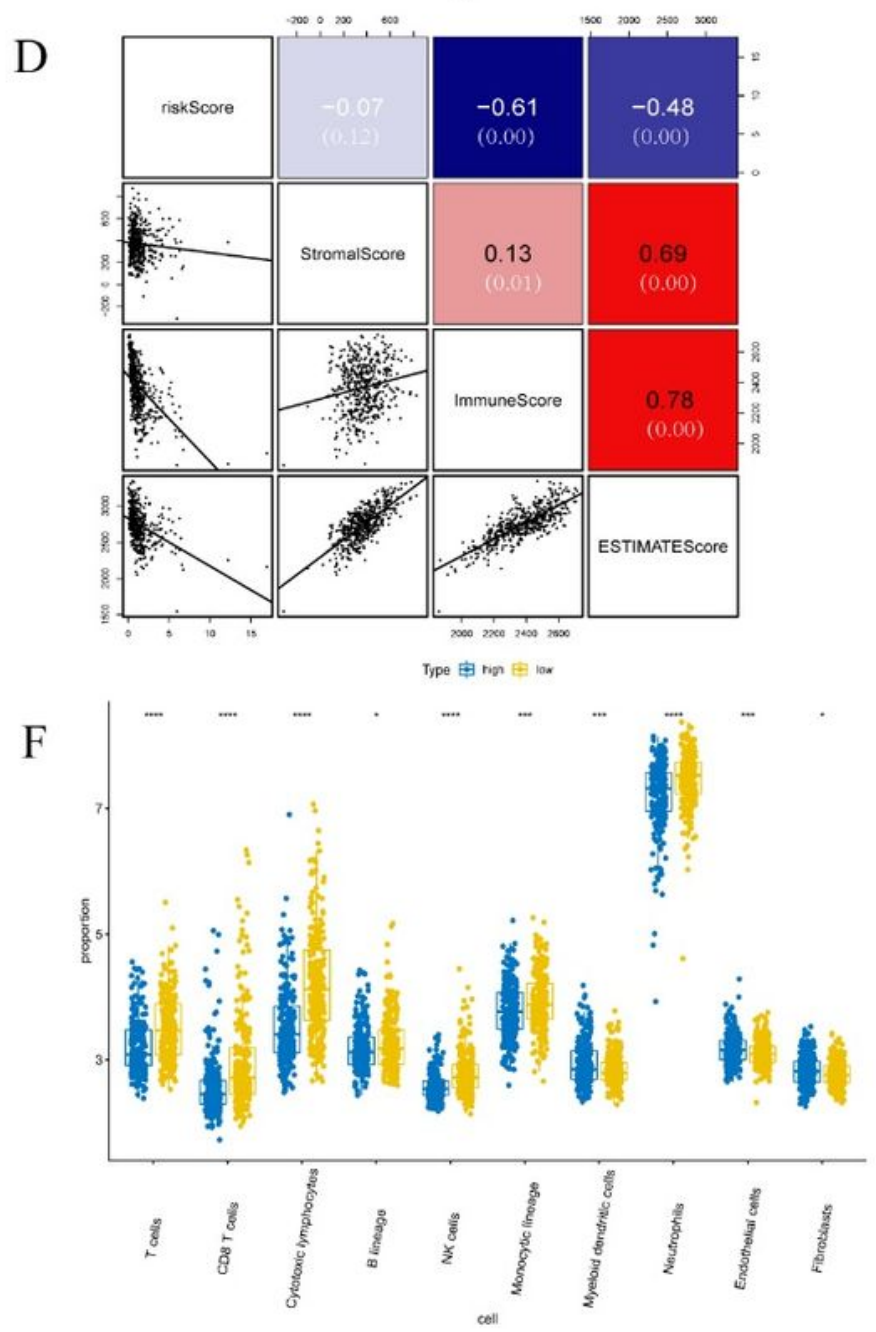

Figure 5

Immune microenvironment of high a nd low risk groups (Boxplot of stromal score in high and low risk group $s(P<05) 05)$. (Boxplot of immune score in high and low risk groups $(P<0.05)$. (Boxplot of ESTIMATES score in high and low risk group $s(P<0.05)$. (D) Scatterplot and heatmap of correlation between risk score and stromal score, immune score and ESTIMATES score. (Heatmap of immune cells 
calculated by MCP counter . F) Boxplot of immune cells in high and low risk group * represents $p<0.05$, ** represents $p<0.01$, $\star \star \star$ represents $p<0.001 \nabla^{\star \star \star \star *}$ represents $p<0.0001$.

A Type 审 high low

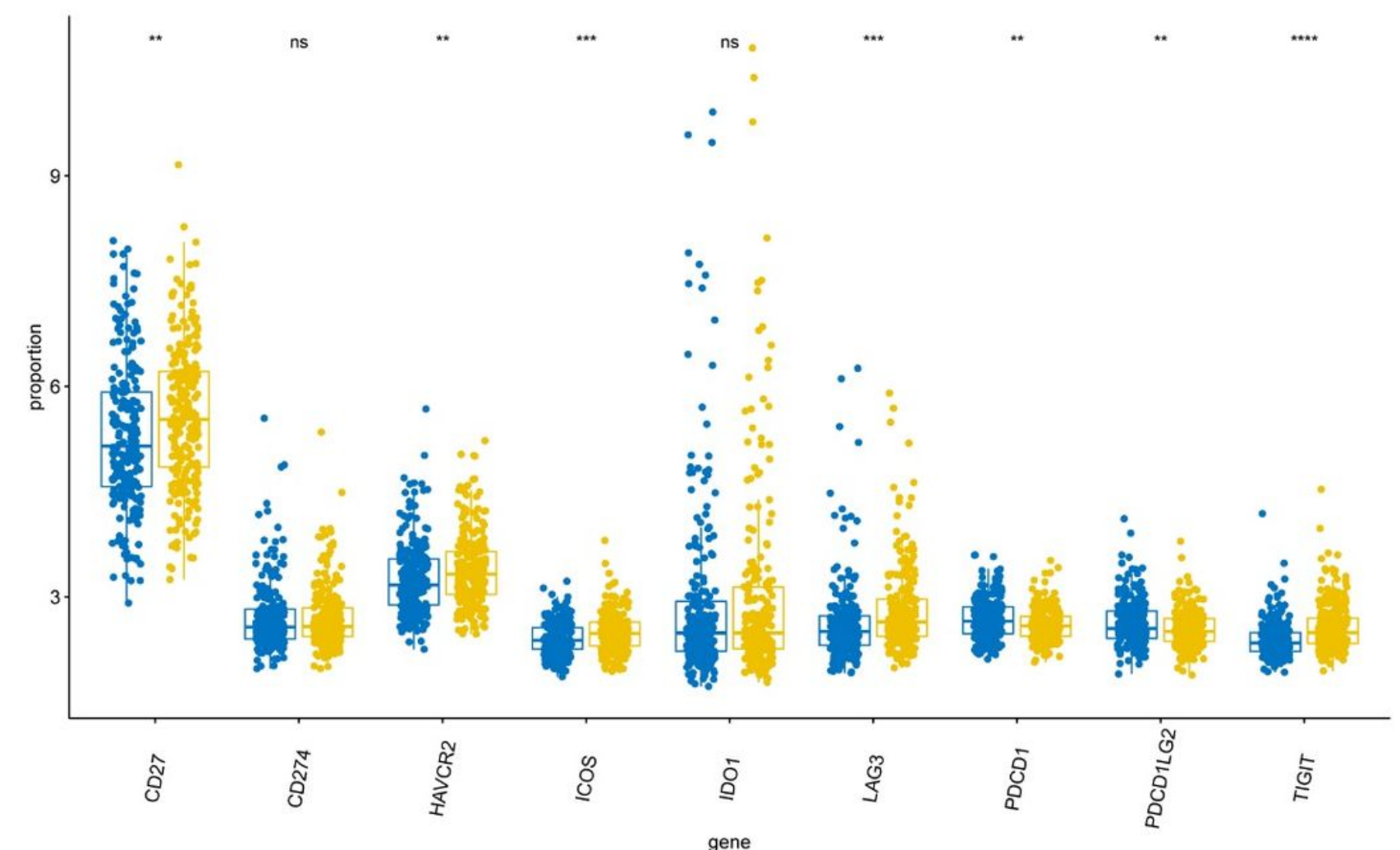

B

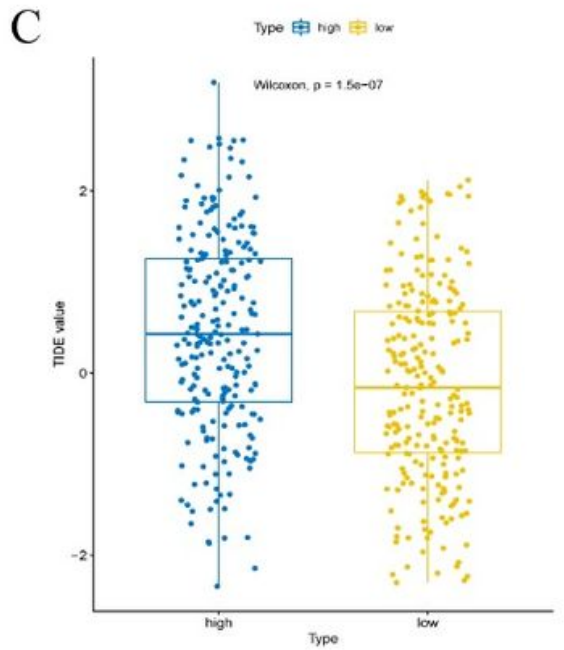

Figure 6

Expression of immune checkpoint molecule and immunotherapy response of $t$ he high and low risk groups. ( Boxplot of immune checkpoint molecules in high and low risk groups ns represents no

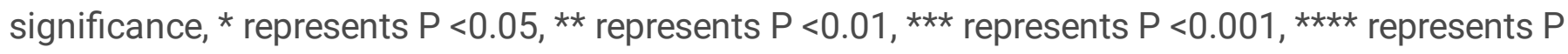
$<0.0001$. (Submap algorithm manifested that low risk group could be more sensitive to the programmed cell death protein 1 (PD 1) inhibitor (Bonferroni corrected P 0.05 C) Boxplot of TIDE score in high and low 
risk groups . The Boxplot showed that the TIDE score of low risk group was lower (Wilcoxon $P=1.5 e$ 07)07), indicating that the low risk group was more sensitive to immunotherapy.

A

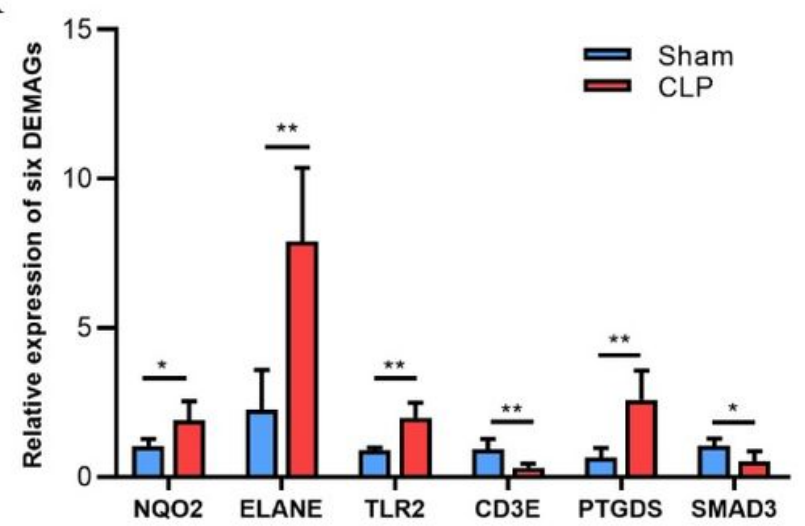

B

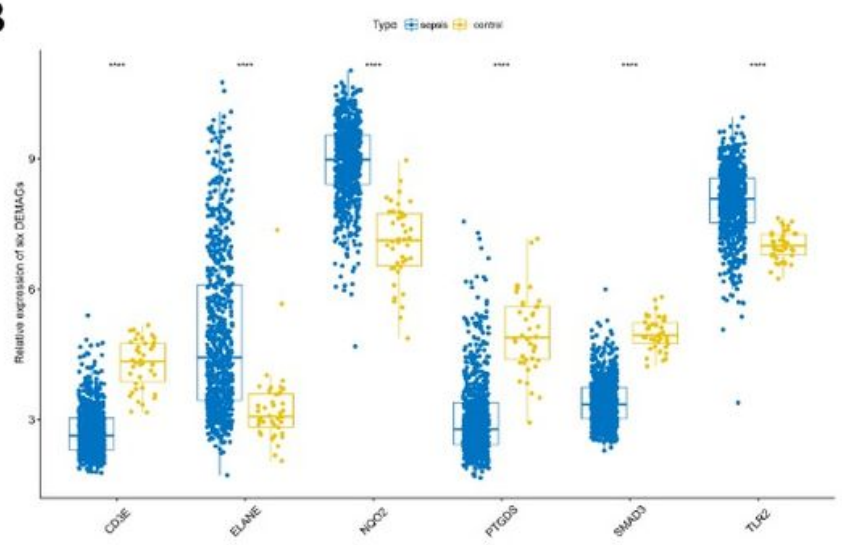

$\mathrm{C}$

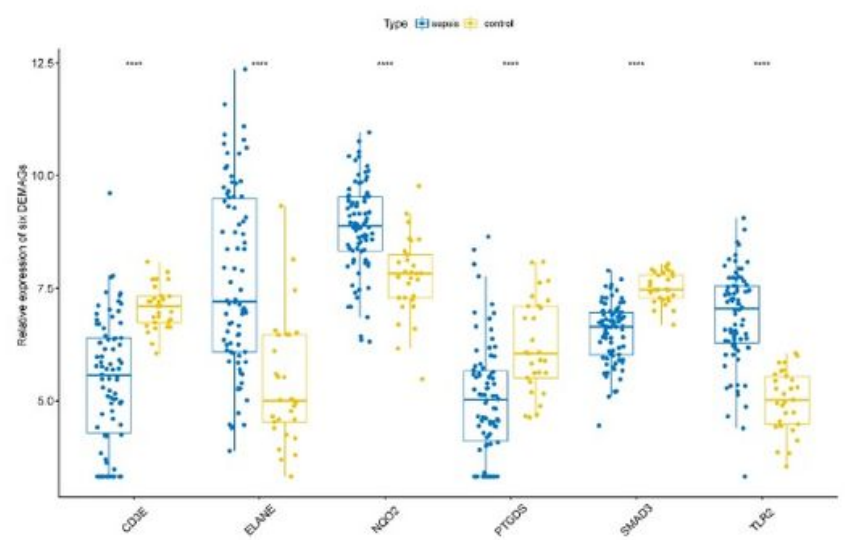

Figure 7

Expression of six DEMAGs in sepsis mice model and two datasets ( Expression levels of DEMAGs in GSE65682 in mice model determined by quantitative real time PCR. Sham: The control group mice constructed by sham operation $n=5$; CLP: Sepsis mice model contrasted by ceca I ligation and puncture $\mathrm{n}=5$. (B) Expression levels of DEMAGs in GSE65682. (C) Expression levels of DEMAGs in GSE69528.

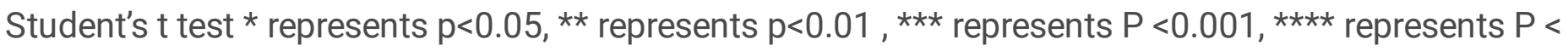
DEMAG s : Differentially expressed metabolism associated genes

\section{Supplementary Files}

This is a list of supplementary files associated with this preprint. Click to download.

- TableS1.xlsx

- Tables2.csv

- TableS3.csv

- Tables4.xls

- Tables5.xls 
- Tables6.xls

- TableS7.xIsx

- Tables8.xIsx 